\title{
Pacific
}

Journal of

Mathematics

\section{GENERIC 8-DIMENSIONAL ALGEBRAS WITH MIXED BASIS-GRAPH}

\author{
THIERRY DANA-PICARD
}




\title{
GENERIC 8-DIMENSIONAL ALGEBRAS WITH MIXED BASIS-GRAPH
}

\author{
ThIERRY DANA-PicARd
}

\begin{abstract}
Deformation theory is the appropriate tool for describing the irreducible components of the scheme $\mathrm{Alg}_{n}$ which parametrizes the structures of $n$-dimensional associative algebras with unit. Each component is "dominated" by one generic or quasi-generic algebra or family of algebras (genericity means that the algebra or the family has only trivial infinitesimal deformations, and quasi-genericity means that the algebra or the family has non trivial infinitesimal deformations, but no algebraic deformation). The components dominated by a generic algebra (or family) are reduced, while the components dominated by a quasi-generic family are non reduced. The invariants we use for that classification are the basis-graph, both weighted and unweighted, of an associative algebra. In this paper, we classify the 8-dimensional algebras with mixed basis-graph and give lower bounds for the numbers of irreducible components of the scheme $\mathrm{Alg}_{8}$, reduced and non reduced.
\end{abstract}

I. Introduction. This paper is a new contribution to the question treated in previous works ([Ha], [Ma], [DP1], [DP2]), namely the study of the irreducible components of the scheme $\mathbf{A l g}_{n}$ which parametrizes the structures of $n$-dimensional associative algebras with unit. The importance of this question was put forward by Gabriel (see [Ga]). In [DP2], the author proved some general lemmas, which enable us to construct deformations of $n$-dimensional algebras from deformations in dimension less than $n$. As already known from [Ha] and [DP2], the main tool is M. Gerstenhaber's theory of deformations of algebras, using Hochschild cohomology (cf. [Ge]) and the appropriate invariants for the classification work are the basis-graph and the weighted basisgraph, as defined in [Sc1] by M. Schaps. In dimension equal to or greater than 6 , the task of writing down a complete deformation chart is unilluminating, as the number of different isomorphism classes of algebras increases very fast, but it is still possible to determine good lower bounds for the number $p(n)$ of reduced irreducible components and the total number $q(n)$ of irreducible components of $\mathbf{A l g}_{n}$, including the non reduced ones.

In $\S I I$, we recall the main definitions and theorems, without proof, from the theory of deformations of associative unitary algebras; the 
proofs are to be found in [Ge], [Sc1], [DP1], [DP2].

In $§ I I I$, we give a complete list of candidates for genericity with mixed 8-dimensional basis-graph and explain for each one either why it is generic (resp. quasi-generic) or how it deforms.

As in [DP1], [DP2], we used the computer program described in [DP-Sc4], which computes, among other things, $Z^{2}(A, A), H^{2}(A, A)$ and $\operatorname{dim}(\operatorname{Aut} A)$, for a given algebra $A$. For 5-dimensional algebras, we still use Happel's notation in [Ha].

II. Basis-graphs and deformations. Denote by $\mathbf{A l g}_{n}$ the structure constant scheme for associative unitary algebras of dimension $n$ over an algebraically closed field $\mathbf{K}$ of characteristic 0 or $\gg 0$. Since the defining equations of the scheme are defined over $\mathbf{Z}$, there are only a finite number of primes for which the number and nature of irreducible components in $\operatorname{Alg}_{n}(\mathbf{K})$ will be different from the characteristic 0 case. Thus any characteristic which is sufficiently large relative to $n$ will give the same classification.

The linear group $\mathrm{GL}(n, \mathbf{K})$ operators on $\mathbf{A l g}_{n}$ by "basis change" (cf. [Kr]): the orbits of $\operatorname{Alg}_{n}$ under the action of $\mathrm{GL}(V)$ correspond to the isomorphism classes and the stabilizer of an algebra $A$ is its automorphism group Aut $A$ (of course automorphisms as an algebra).

Definition. If an algebra or an algebraic family of algebras $A$ lies in the closure of the orbit of a different algebra or family $A^{\prime}$, we will say that $A$ is a specialization of $A^{\prime}$ or that $A^{\prime}$ is a deformation of $A$. The fact that $A^{\prime}$ is a deformation of $A$ will be represented by $A^{\prime} \rightarrow A$.

There is another definition of deformations, that we will use as well:

Definition. Let $\left(C, t_{0}\right)$ be any pointed scheme, with $C=\operatorname{Spec}(R)$ for $R$ a commutative affine ring over $\mathbf{K}$ and $t_{0}$ a closed point corresponding to a maximal ideal $\mathfrak{m}_{0}$ of $R$. A flat deformation $B$ of $B_{0}$ over $\left(C, t_{0}\right)$ is a flat $R$-algebra $B$ together with an isomorphism of $B_{0}$ with $B \otimes_{R} R / \mathfrak{m}_{0}$. When the fibers over general closed points of $C$ are all isomorphic to an algebra $A$, then $A$ is also called a deformation of $B_{0}$.

If $C$ is an irreducible algebraic scheme of dimension at least one, the deformation is called algebraic; if $R=\mathbf{K}[\varepsilon] /\left(\varepsilon^{2}\right)$, the deformation is a first order deformation. In general, if $\operatorname{dim} C=0$, the deformation is called infinitesimal.

Definition. (1) An algebra is rigid if its orbit under the action of $\mathrm{GL}(n)$ is dense in an irreducible component of $\mathbf{A l g}_{n}$. (2) A family of 
algebras is semi-rigid if the union of the orbits of the algebras in this family, under the action of base changes, is dense in an irreducible component of $\mathbf{A l g}_{n}$.

In both of these cases, we will say that the algebra (or the family of algebras) is generic. An algebra or family giving the reduced part of a non reduced component will be called quasi-generic. (A family is quasi-generic if it has a non trivial first order deformation, but no algebraic deformation.)

It is important to note that this definition of genericity and quasigenericity is not in conflict with the traditional definition of genericity: as the field $K$ is algebraically closed, a single algebra over an extension of $K$ is the same as a parametrized family of algebras over $K$, and a variety is defined by its algebraic points.

The basis-graph of a finite dimensional algebra $A$ is the diagram constructed as follows:

(i) Take a number of vertices equal to the number of idempotents in a complete set of primitive orthogonal idempotents $\left\{e_{i}\right\}$ and label each vertex by an idempotent.

(ii) For $i$ not equal to $j$, the number of arrows from $e_{i}$ to $e_{j}$ is equal to $\operatorname{dim}\left(e_{i} A e_{j}\right)$.

(iii) The number of loops from $e_{i}$ to itself is equal to $\operatorname{dim}\left(e_{i} A e_{i}\right)-$ 1 .

We get the weighted basis-graph of $A$ by adding weightings on the arrows in the following way:

(iv) The number of arrows from $e_{i}$ to $e_{j}$ with weight $k$ is $n_{i j}^{k}=$ $\operatorname{dim} e_{i}\left(J^{k} / J^{k+1}\right) e_{j}$, where $J$ is the radical of $A$; a $k$-weighted arrow is an arrow with $k$ barbs.

(v) Matrix units are weighted by $\infty$ and marked by a solid triangular barb.

Let $e$ be a primitive idempotent in the algebra $A$. A loop $x \in e A e$ is trivial if its products with all basis elements of $A$, different from $e$, are zero. A trivial loop will be denoted by $\&$ and has weight 1 .

EXAMPLES. The weighted basis-graphs in Figure 1 represent the following algebras:

(a) the 4-dimensional Kronecker algebra;

(b) $M_{2}(\mathbf{K})$;

(c) the algebra of $3 \times 3$ upper triangular matrices;

(d) $\mathbf{K}[x] /\left(x^{3}\right)$;

(e) $F^{4}=\mathbf{K}\langle x, y\rangle /\left(x^{2}, y^{2}, y x-\sigma x y\right)$, with $\sigma \neq-1,0,1$;

(f) $\mathbf{K}[x, y] /(x, y)^{2}$. 


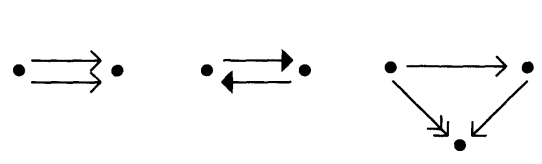

(a)

(b)

(c)

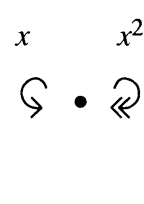

(d)

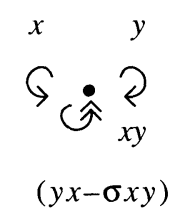

(e) $x \quad y$

$\Leftrightarrow$ -

(f)

Figure 1

NotATIONS. In what follows, we will generally give results in tabular forms, namely "idempotents-filtered radical basis-relations", where $J$ denotes the radical of the algebra. As an example, let us translate some of the basis-graphs in Figure 1:

(a) $e_{0}, e_{1} ; x_{1}, x_{2} \in e_{0} J e_{i}$.

(c) $e_{0}, e_{1}, e_{2} ; x_{1} \in e_{0} J e_{1}, x_{2} \in e_{1} J e_{2}, x_{1}, x_{2} \in e_{0} J e_{2}$.

(e) $e_{0} ; x, y, x y \in e_{0} J e_{0} ; y x=\sigma x y$ ( $\sigma$ different from $\left.-1,0,1\right)$.

On these weightings, we define a partial ordering: suppose $\Phi$ and $\Phi^{\prime}$ are two weightings on the same basis-graph $Q$. Then $\Phi \leq \Phi^{\prime}$ if for each pair $i, j$ and for each natural number $k$, the number of arrows from $i$ to $j$ of weight greater than or equal to $k$ in $\Phi$ is less than or equal to the corresponding number in $\Phi^{\prime}$.

EXAMPLES. (1) The weightings of the basis-graphs in Figure 2 cannot be compared, despite the fact that the basis-graphs are identical:
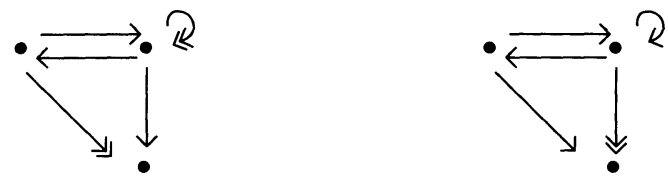

Figure 2

(2) The local algebra $\mathbf{K}[x, y] /(x, y)^{2}$ deforms to $\mathbf{K}[z] /\left(z^{2}\right)$, the deformation being given by: $x=t\left(z+z^{2}\right), y=t\left(z-z^{2}\right)$. The corresponding basis-graphs are (f) and (d) in Figure 1.

These graphs are the appropriate invariants for the classification work. In fact, if $A^{\prime}$ is a deformation of $A$, then the basis-graph of $A$ either equals the basis-graph of $A^{\prime}$, or is obtained by coalescing vertices, replacing each vanishing vertex by a loop. This preserves the dimensions in the Peirce decomposition of $A$. 
These graphs have the suitable semi-continuity properties as requested by $\mathbf{M}$. Gerstenhaber in [Ge]; in particular, on the weightings of a given basis-graph a partial ordering compatible with specialization can be defined. For studying genericity we will need to consider only maximal weightings. (cf. [DP], [DP-Sc1]).

In [Sc1], M. Schaps defined the basis-graph as an extension of the quiver. Here, as in [DP2], in order that the composition of arrows should appear in a natural order corresponding to the multiplication in the algebra, we dualize the definition. As far as the classification is concerned, this dualization is irrelevant, since an algebra is generic if, and only if, its dual is generic.

LEMMA [DP-Sc1]. $A$ set of $n$ vertices in a basis-graph algebra $A_{Q}$ can be deformed to a matrix block if and only if they are connected, they are completely symmetric with regard to all permutations, and the number of loops at each vertex is one less than the number of arrows from each point to another. In particular, a matrix block configuration must contain a two-arrow cycle.

In order to find the candidates for genericity and to check their actual genericity, we act as follows: we determine the type-generic algebras or families, i.e. the "most generic" algebras with a given basisgraph, and then, generally by a computer calculation, we determine $H^{2}(A, A)$. If it is zero for an algebra $A$, then the orbit of $A$ is dense in the component containing $A$ and $A$ is rigid (cf. [Ge]). For a family of algebras, in order to prove semi-genericity or to show that a first order deformation is associative, it suffices to look at special values of the parameters; if for an algebra $A$ in the family the dimension of $H^{2}(A, A)$ is equal to the number of parameters in the family, then the family is semi-rigid (cf. [DP-Sc2]).

The problem of proving that a type-generic component is not generic is more difficult. If $W$ contains an open dense orbit, and for some algebra $A$ in this orbit, $H^{2}(A, A)$ is not zero, then the algebra is not rigid. It may, however, represent a generic closed point in a nonreduced component of $\mathbf{A l g}_{n}$. When $W$ does not have a single orbit, but must be given by a family, the situation is yet more difficult, for in order to demonstrate that this family is not semi-rigid, it is necessary to construct an infinitesimal deformation which is valid for all values of the parameters in an open dense set. This situation already appeared in dimension 6 with one family with mixed basis-graph and permitted us to show that $\mathbf{A l g}_{n}$ has non reduced components for all 
$n \geq 6$ (cf. [DP2], [DP-Sc3]), but since the family has a single parameter the deformation was not difficult to construct.

III. Construction of deformations in higher dimensions. In low dimension, it is possible to write down lists of algebras and to check them for genericity. As the dimension increases, it is necessary to get some general results in order to eliminate most cases as quickly as possible.

It is obvious that adding a trivial loop on one idempotent of the basis-graph of the algebra $A$ (with radical $J$ ) determines a new algebra $A[z] /\left(z^{2}, z J, J z\right)$, which is trivially deformable.

If $A_{1}^{\prime}$ and $A_{2}^{\prime}$ are respective deformations of $A_{1}$ and $A_{2}$, then $A_{1}^{\prime} \times A_{2}^{\prime}$ is clearly a deformation of $A_{1} \times A_{2}$; therefore we will be mainly interested in "connected" algebras.

An algebra $A$ is called basic if it contains no total matric subalgebra. An idempotent of an algebra $A$ is called basic if it does not belong to a total matric subalgebra of $A$.

Let $A$ be an algebra and $S$ a complete primitive decomposition set which spans $A / \operatorname{Rad}(A)$; recall that $A / \operatorname{Rad}(A)$ is a direct sum of matrix blocks. Let $S^{\prime}=\left\{f_{1}, \ldots, f_{r}\right\}$ be a subset of $S$ formed by choosing one idempotent in each matrix block and set $f=f_{1}+\cdots+f_{r}$. The algebra $\bar{A}=f A f$ is called the skeleton of $A$ (up to isomorphism, the skeleton of $A$ is independent of the choice of $S^{\prime}$ ). Algebras with the same skeleton are called Morita equivalent. It is well known that two Morita equivalent algebras have the same representation theory; therefore representation theory can be done with basic algebras. M. Schaps proved in [Sc1] that the same holds for deformation theory, namely that the algebras appearing in deformations of an algebra $B_{0}$ are in one-to-one correspondence with those appearing in deformations of the skeleton $\bar{B}_{0}$. As a consequence, if the respective dimensions of $B_{0}$ and $\bar{B}_{0}$ are $n$ and $p$, one may naturally identify the components of $\mathbf{A l g}_{n}$ containing $B_{0}$ with those of $\operatorname{Alg}_{p}$ containing $\bar{B}_{0}$.

The following lemmas and propositions are proven in [DP2]:

Let $A_{1}$ and $A_{2}$ be two algebras, and $e_{1} \in A_{1}, e_{2} \in A_{2}$ be primitive basic idempotents. Let $N_{1}, N_{2}$ be ideals of $A_{1}, A_{2}$ respectively, such that $A_{1} / N_{1} \stackrel{\sim}{\rightarrow} K$ has the residue of $e_{1}$ as identity. The fiber product $A_{1} \times A_{2}$ of $A_{1}$ with $A_{2}$ along $\left(e_{1}, e_{2}\right)$ is the set of all elements in $A_{1} \times A_{2}$ such that both components have the same residue modulo $N_{1} \times N_{2}$.

We denote by $T$ the algebra of $2 \times 2$ upper triangular matrices. As its basis-graph is $e_{1} . \rightarrow . e_{0}$, we'll call $T$ the arrow-algebra. 
LeMmA 1. Let $A$ be an algebra with primitive basic idempotent $e$, and $B$ the algebra which is the fiber product of the arrow-algebra $T$ with $A$ along $\left(e_{0}, e\right)$. The deformations of $B$ are completely determined by those of $A$, i.e. if $B^{\sim}$ is a deformation of $B$, then $B^{\sim}$ is the fiber product of $T$ with $A^{\sim}$ along $\left(e_{0}, e^{\sim}\right)$, where $A^{\sim}$ is a deformation of $A$ and $e^{\sim}$ is a basic primitive idempotent of $A^{\sim}$.

REMARKs. (1) By duality, the corresponding result is evidently true for the fiber product $T \times A$ along $\left(e_{1}, e\right)$.

(2) This lemma is a major component of the proof that $\mathbf{A l g}_{n}$ is non reduced for $n \geq 6$ (cf. [DP-Sc3]).

Let $S=\left\{e_{1}, \ldots, e_{r}\right\}$ be a complete set of orthogonal idempotents of an algebra $A$. Following M. Schaps, we say that a basis $B=\left\{x_{1}, \ldots, x_{n}\right\}$ of $A$ respects $S$ if for every $i \in\{1, \ldots, r\}$, $x_{i}=e_{i}$, and if every $x_{i}$ lies in a component $e_{i} A e_{j}$ of the Peirce decomposition, $A=\bigoplus e_{i} A e_{j}$.

LEMMA 2. Let $A$ be a basic algebra with a complete orthogonal idempotent set $e_{1}, \ldots, e_{r}, A^{\sim}$ be a deformation of $A$ and $x_{1}, \ldots, x_{n}$ be a basis for $A^{\sim}$ which respects the idempotent set $e_{1}^{\sim}, \ldots, e_{r}^{\sim}$. Let $\left\{f_{i j}, 1 \leq i \leq r, 1 \leq j \leq r\right\}$ be a complete orthogonal idempotent set for $A^{\sim}$ such that $\sum_{j} f_{i j}$ is a lifting of $e_{i}$. If $f_{i j}$ is basic in $A^{\sim}$ and if $x_{q}$ is a basis element contained in $e_{i}^{\sim} A^{\sim} e_{i}^{\sim}$, then the coefficient of $f_{i j}$ in $x_{q}$ lies in $\mathfrak{m}_{0}$ (where $\mathfrak{m}_{0}$ is the distinguished maximal ideal of the parameter ring $R$ ).

Proposition 1. Let $A^{\sim}$ be a deformation of the algebra $A, f_{i j}$ be a primitive idempotent which is defined at the general fiber of $A^{\sim}$. Let $B^{\sim}$ be an algebra which contains $A^{\sim}$ as a subalgebra, in such a way that a basis for $B^{\sim}$ is obtained by adding to a basis of $A^{\sim}$ containing $f_{i j}$ exactly one idempotent $f$ for which $\operatorname{dim} f_{i j} B^{\sim} f=p$, $\operatorname{dim} f B^{\sim} f=1$ and every other Peirce component either starting or ending at $f$ is equal to 0 . Then $B^{\sim}$ is a deformation of the algebra $B$, the fiber product of the algebra $\mathrm{Kr}_{p}$ with $A$ along $\left(e, e_{1}\right)$ where $e$ is the right idempotent of the Kronecker algebra $\mathrm{Kr}_{p}$. Note that reversing the arrows of $\mathrm{Kr}_{p}$, we get the dual result.
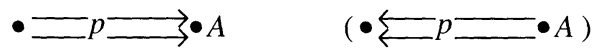
Proposition 2. Let $A$ be a local algebra having a deformation $A^{\sim}$ whose fibers are local algebras. Let $J$ be the radical of $A$, and let $I$ be an ideal of codimension $\leq 1$ in $J$. Let $B$ be an algebra with two idempotents $e$ and $f$, two arrows $z$ and $w$ from $e$ to $f$ and $f B f=A$. Suppose that $w \cdot J=0$, that $z \cdot J$ is contained in $\langle w\rangle$ and that $I$ is the annihilator of $z$ in $J$. Then $B$ has a non trivial deformation $B^{\sim}$ with two idempotents $e^{\sim}, f^{\sim}$, two arrows $z^{\sim}$ and $w^{\sim}$, and $f^{\sim} B^{\sim} f^{\sim}=A^{\sim}$.
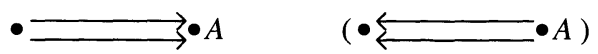

The deformations given here are not idempotent-splitting; in the case $\operatorname{codim}_{J} I=1$, they require that $A$ has a non trivial local deformation. We will see further that we get a non trivial idempotentsplitting deformation in the case where $A$ has a trivial loop.

As a consequence, given an algebra $B$ as in Proposition 2, we need only consider it a candidate for genericity if $A$ has no local deformation preserving the socle dimension and no trivial loop.

Definition. An idempotent $e$ in an algebra $A$ is called loopless if $\operatorname{dim} e A e=1$, i.e. there is no loop on the basis-graph at that point.

LEMMA 3. Let $A_{1}$ and $A_{2}$ be connected algebras consisting of more than one point, $e_{1} \in A_{1}$ and $e_{2} \in A_{2}$ primitive basic loopless idempotents. Then the deformations of the fiber product $B$ of $A_{1}$ and $A_{2}$ along $\left(e_{1}, e_{2}\right)$ correspond to pairs of deformations $\left(A_{1}^{\sim}, e_{1}^{\sim}\right)$ and $\left(A_{2}^{\tilde{2}}, e_{2}^{\sim}\right)$ of $\left(A_{1}, e_{1}\right)$ and $\left(A_{2}, e_{2}\right)$ such that $e_{1}^{\sim}$ and $e_{2}^{\sim}$ are still basic.

Corollary (one-way bridge). Let $A$ be an algebra whose basisgraph can be represented by $A_{1} \bullet=p \rightrightarrows \bullet A_{2}$, where the points represent loopless idempotents and $=p \longrightarrow$ means that there are $p$ arrows in the same direction. Then every deformation of $A$ has a basis-graph of the form $A_{1}^{\sim}=p \Longrightarrow A_{2}^{\sim}$, where $A_{1}^{\sim}$ is a deformation of $A_{1}$.

Lemma 4. Let $A_{1}, A_{2}$ be two connected algebras and $e_{i} \in A_{i} \quad(i=$ $1,2)$ two basic primitive idempotents, possibly looped. If $A_{1}$ and/or $A_{2}$ have basic deformations, then the fiber product $A_{1} \times A_{2}$ along $\left(e_{1}, e_{2}\right)$ is deformable.

Corollary. Let $Q$ be a basis-graph, let $f_{i}$ be an idempotent. Suppose that $f_{i}$ is loopless and is connected to all other idempotents by 
at most one arrow. Then the local ring at $f_{i}$ for any algebra with basis-graph $Q$ deforms independently of the remainder of the algebra. In particular, if there are exactly one or two loops at $f_{i}$, then every algebra with that basis-graph has idempotent-splitting deformations.

Definition. A connected basis-graph with two idempotents $e_{0}, e_{1}$ has 2-type $(a, b, c, d)$ when $a=\operatorname{dim} e_{0} A e_{0}-1, b=\operatorname{dim} e_{0} A e_{1}$, $c=\operatorname{dim} e_{1} A e_{0}$ and $d=\operatorname{dim} e_{1} A e_{1}-1$.

LEMMA 5. Let $A$ be an algebra with a basis-graph containing exactly two idempotents and of 2-type $(1,0, c, 0)$ or $(1, c, 0,0)$, where $c$ is a non zero integer. Then $A$ has an idempotent-splitting deformation.

LEMMA 6. (i) If $A$ is a local algebra and $B$ is a mixed algebra with two idempotents $e_{0}, e_{1}$ such that $e_{0} B e_{0} \stackrel{\sim}{\rightarrow} A$ and $\operatorname{dim}\left(e_{i} B e_{j}\right)=1$ for $i, j$ not both zero, then the product of the two arrows in $B$ must lie in the socle of $A$.

(ii) Let $A$ be a local algebra having a local deformation $A^{\sim}$ such that the dimension of the socle is fixed. We define an algebra $B$ with mixed basis-graph as in (i); then $B$ has a deformation.

-

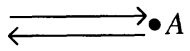

LEMMA 7. Let $A$ be a local algebra whose socle is not contained in $(\operatorname{Rad} A)^{2}$ and let $B$ be an algebra having a basis containing two idempotents $e_{1}, e_{2}$ such that the local ring at $e_{1}$ is isomorphic to $A$, $\operatorname{dim} e_{2} B e_{1}=2, \operatorname{dim} e_{1} B e_{2}=0$ and $\operatorname{dim} e_{2} B e_{2}=1$. Then $B$ has an idempotent-splitting deformation.

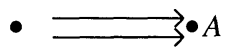

Proposition 3. Let $A^{\prime}$ be an algebra with mixed basis-graph containing exactly two idempotents $e_{0}, e_{1}$ such that $\operatorname{dim} e_{1} A^{\prime} e_{1}=1$. Suppose $A^{\prime}$ contains an arrow $x$ of $e_{1} A^{\prime} e_{0}$ such that $x \notin\left(\operatorname{Rad} A^{\prime}\right)^{2}$ and $x$ lies in the socle, so that $A^{\prime}(x) A^{\prime}$ is an ideal of dimension 1 . Let $A=A^{\prime} /(x)$. Then if $A$ has a basic deformation, so does $A^{\prime}$.

\section{The classification in dimension 8.}

Notation. In the case by case study, we denote the given algebra by $B_{0}$ and the deformed algebra by $B$. 
We give now the list of 8-dimensional mixed basis-graphs which are candidates for genericity, organized according to the number of idempotents, and when there are two idempotents, according to the 2-type. Since the list of all possible mixed basis-graphs is considerably longer in dimension 8 than in every lower dimension, we eliminate those basis-graphs with trivial deformations before writing the list. The algebras listed as candidates for generic algebras have no trivial loops and are locally maximally weighted (see [DP2]); in general, we will not give the proof of local maximal weighting when it follows from lemmas already proven. Furthermore, we will freely use the trivial automorphisms multiplying basis elements by constants, in order to eliminate superfluous parameters from the defining equations of algebras with mixed basis-graph.

We indicate by $(*)$ the algebras or families which will be shown to be generic and by $(* *)$ the families which will be shown to be quasi-generic.

The 8-dimensional generic (resp. quasi-generic) algebras and families of algebras $B_{0}$ with mixed basis-graph can be classified into three classes:

(1) $B_{0}=\mathbf{K} \times A^{\prime}$, where $A^{\prime}$ is a 7-dimensional generic (resp. quasigeneric) algebra or family of algebras. There are 10 (resp. 5) such algebras or families (cf. [DP1]); note that this class contains the algebras of the form $\mathbf{K}^{2} \times A^{\prime \prime}$, where $A^{\prime \prime}$ is 6-dimensional and generic (resp. quasi-generic) with mixed basis-graph.

As there is neither a connected loop-only nor a connected mixed algebra of dimension 5 which is generic (resp. quasi-generic), there is no need to consider products $A_{1} \times A_{2}$ where $A_{1}$ is 5-dimensional and $A_{2}$ is either the arrow-algebra $T$ or $\mathbf{K}^{3}$. For similar reasons, the case where $A_{1}$ is 3-dimensional looped and $A_{2}$ is 5-dimensional loopless contains no generic algebra.

(2) $B_{0}=A_{1} \times A_{2}$, where $A_{1}$ is the 4-dimensional semi-rigid family and $A_{2}$ is either $M_{2}(\mathbf{K})$ or the 4-dimensional Kronecker algebra. These two cases are generic.

(3) What remains to consider are the "connected" algebras. Let us list the connected basis-graphs with their maximal weightings:

(a) Let $B_{0}$ be an algebra with basis-graph of 2-type $(1,1,0,4)$ (resp. $(2,1,0,3))$. By Lemma $5, B_{0}$ has an idempotent-splitting deformation.

(b) Let $B_{0}$ be an algebra with basis-graph as in Figure 3a, where the line represents an arrow in any direction and $A$ a mixed or local 
6-dimensional algebra. The algebra $B_{0}$ is generic or quasi-generic whenever $A$ is generic or quasi-generic, by Lemma 1 (such an algebra can be quasi-generic when $A$ is generic).

(c) Let $B_{0}$ be an algebra with basis-graph as in Figure 3b, where $A$ is mixed 5-dimensional, $A$ and its attaching idempotent not looped; as there is no 5-dimensional mixed generic algebra, by Proposition 1 every algebra with such a basis-graph is non generic.

(d) If $B_{0}$ is an algebra with basis-graph as in Figure $3 \mathrm{~b}$, where $A$ is local 5-dimensional, we distinguish two cases: the first one when both arrows are in the same direction, the second one when they are reversed.

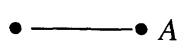

(a)

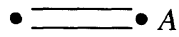

(b)

FIGURE 3

In the first case, by Proposition 1 , every algebra $B_{0}$ has an idempotent-splitting deformation (recall that every type-generic local algebra of dimension 5 has an idempotent-splitting deformation; cf. [Ha]). The second case will be treated in detail below.

(e) Two idempotent algebras not included in (a), (b), (c), (d).

(f) Three idempotent algebras not included in (b).

The algebras requiring detailed study are thus the algebras in the second case of (d), which we will call "reversed arrow algebras", the two idempotent algebras in (e) and the three idempotent algebras in (f). We dedicate a separate section to each reversed arrow algebra.

Denote the orthogonal primitive idempotents by $e_{0}, e_{1}$ and the arrows by $x \in e_{0} J e_{1}, y \in e_{1} J e_{0}, e_{1}$ being the idempotent of $A$. If $y x=0$, as $A$ deforms, the two arrows don't interfere in the deformation, as the algebra can be considered as the fiber product of the 4-dimensional 2-arrow cycle algebra with a deformable 5-dimensional local algebra, and $B$ clearly deforms (in a way similar to what happens in the previous case). Let's study the case where $y x$ is not zero. We have the following subcases; by Lemma 6, we know that these are all the weightings which occur (recall that a Scorza $(c, d)$ algebra is a local algebra with radical $J$ such that $\operatorname{dim} J / J^{2}=c$ and $\operatorname{dim} J=c+d)$. 
(i) $A$ is a Scorza $(3,1)$-algebra. Every Scorza $(3,1)$-algebra deforms into Happel's $B_{s}^{5}$ family, i.e. $B_{s}^{5}$ is the Scorza $(3,1)$ typegeneric family. Therefore, by Lemma 7 , it suffices to consider only the case where $A=B_{s}^{5}$. As a help to the reader in translating, we will write down the deformation both in graphic (Figure 4) and tabular form:
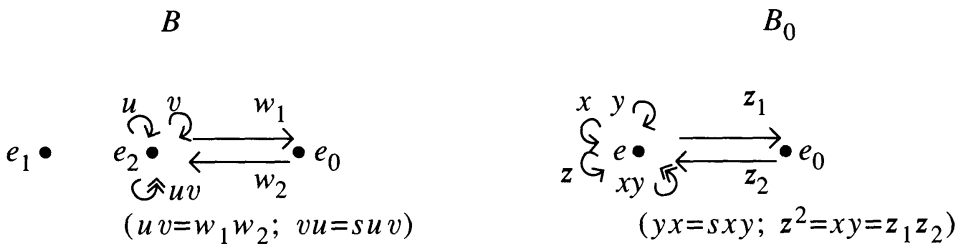

FIGURE 4

$B_{0}: e_{0}, e ; x, y, z, x y \in e_{0} J e_{0} ; z_{1} \in e_{0} J e_{1} ; z_{2} \in e_{1} J e_{0} ; y x=$ $\sigma x y(\sigma$ distinct from $-1,0,1), z^{2}=x y=z_{1} z_{2}$.

$B: e_{0}, e_{1}, e_{2} ; u, v, u v \in e_{2} J e_{2}, w_{1} \in e_{2} J e_{0}, w_{2} \in e_{0} J e_{2} ; w_{1} w_{2}$ $=u v, v u=\sigma u v$.

Here $e=e_{1}+e_{2}, x=u, y=v, z=t\left(e_{1}-e_{2}\right)+(1 / 2 t) u v$, $z_{1}=w_{1}, z_{2}=w_{2}$.

For explicit deformations in the subsequent cases, see [DP1]. Let now $A=B_{s}^{8}$ or $A=B_{-1}^{8}$ or $A=B^{10}$ or $A=B_{c}^{10}$.

(1) Suppose that $u_{1} u_{2}=z$. In the above four cases, the resulting algebra is a fiber product of two deformable algebras, hence is itself deformable.

(2) Suppose that $u_{1} u_{2} \neq z$. By Lemma 6, it suffices to study the case where $A=B_{s}^{8}$, as the other algebras are specializations of it (cf. [Ha]). Therefore we consider the algebra $B_{0}$ with basis-graph given by $e_{0}, e_{1} ; x, y, z, x y \in e_{1} J e_{1}, z_{2} \in e_{0} J e_{1}, z_{1} \in e_{1} J e_{0}$, where $y x=s x y$ and $z_{1} z_{2}=\alpha z+\beta x y$. It has the first order deformation defined by the following multiplication table, with $\varepsilon^{2}=0$ :

\begin{tabular}{|c|cccccc|}
\hline & $x_{2}$ & $x_{3}$ & $x_{4}$ & $x_{5}$ & $x_{6}$ & $x_{7}$ \\
\hline$x_{2}$ & & $x_{5}$ & & & & \\
$x_{3}$ & $s x_{5}$ & & & & & \\
$x_{4}$ & & & $\varepsilon x_{4}+\varepsilon(\beta / \alpha) x_{5}$ & & $\varepsilon x_{6}$ & \\
$x_{5}$ & & & & & & \\
$x_{6}$ & & & & & & $\alpha x_{4}+\beta x_{5}$ \\
$x_{7}$ & & & $\varepsilon x_{7}$ & & $\alpha \varepsilon x_{1}$ & \\
\hline
\end{tabular}


It can easily be checked that this multiplication is associative and that, for an algebra $A$ with weighted basis-graph as above, there exists an element of $H^{2}(A, A)$ for which the deformation part of the tensor has non zero inner product with the deformation part of the tensor given by the previous table. Therefore $A$ is not generic, but we do not know whether it has an algebraic deformation or not.

(ii) $A$ is a Scorza $(2,2)$-algebra. For every $A \in \operatorname{Scorza}(2,2)$, there are two loops, say $u_{1}$ and $u_{2}$, of weight 2 ; we have to consider each time three cases (cf. Lemma 6): (1) $z_{1} z_{2}=u_{1}$; (2) $z_{1} z_{2}=u_{2}$; (3) $z_{1} z_{2}=\alpha u_{1}+\beta u_{2}$, where $\alpha$ and $\beta$ are arbitrary parameters; indeed, by a trivial automorphism, we can reduce the study of the case where one of them is equal to 1 , the second one being non zero; note that one of the two first cases is automatically included in this "general" one.

By Lemma 6, the only type-generic family we have to consider here is when $A=C_{s}^{5}$.

(1) We consider the family $B_{0}$ whose weighted basis-graph is generated by two idempotents $e_{0}, e_{1}$ and $x, y, x y, y x \in e_{1} J e_{1}, z_{1} \in$ $e_{1} J e_{0}, z_{2} \in e_{0} J e_{1}$ such that $x^{2}=y x, z_{1} z_{2}=y^{2}=s x y$.

Now let $B^{\sim}$ be the algebra with basis $x_{0}, x_{1}, x_{2}, x_{3}, x_{4}, x_{5}, x_{6}$ and $x_{7}$ and following multiplication table (cf. [Ha], pp. 473-474):

\begin{tabular}{|l|cccccccc|}
\hline & $x_{0}$ & $x_{1}$ & $x_{2}$ & $x_{3}$ & $x_{4}$ & $x_{5}$ & $x_{6}$ & $x_{7}$ \\
\hline$x_{0}$ & $x_{0}$ & & & & & & & \\
$x_{1}$ & & $x_{1}$ & & $x_{3}$ & $x_{4}$ & $x_{5}$ & & \\
$x_{2}$ & & & $x_{2}$ & & & & & \\
$x_{3}$ & & $x_{3}$ & & & & & & \\
$x_{4}$ & & & $x_{4}$ & & & & & \\
$x_{5}$ & & & & & & & $x_{3}$ & $x_{5}$ \\
$x_{6}$ & & $x_{6}$ & & & & & & \\
$x_{7}$ & & & & & & & $x_{6}$ & $x_{7}$ \\
\hline
\end{tabular}

all missing entries being equal to 0 . The weighted basis-graph of $B^{\sim}$ is generated by the idempotents $x_{0}, x_{1}, x_{2}, x_{7}$ and the radical basis $x_{3} \in x_{1} J^{\sim} x_{1}, x_{4} \in x_{1} J^{\sim} x_{2}, x_{5} \in x_{1} J^{\sim} x_{7}$, and $x_{6} \in x_{7} J^{\sim} x_{1}$ with $x_{3}=x_{5} x_{6}$. We now define a base change by the following formulae: $y_{1}=t x_{1}+s x_{3}, y_{2}=t\left(x_{1}+x_{2}\right)+x_{3}+x_{4}, y_{3}=t x_{3}, y_{4}=t\left(x_{3}+x_{4}\right)$, $y_{5}=t x_{5}, y_{6}=x_{6}$ and $y_{7}=x_{7}$. With $y=y_{1}$ and $x=y_{2}$, we get a basis of a family $B$ which is a deformation of the family $B_{0}$.

(2) Consider the following family: 
$B_{0}: e_{0}, e_{1} ; x, y, x^{2}, y^{2} \in e_{0} J e_{0}, z_{1} \in e_{0} J e_{1}, z_{2} \in e_{1} J e_{0} ; x y=$ $s y^{2}, y x=x^{2}$ and $z_{1} z_{2}=x^{2}+\sigma y^{2}$.

As in (1), we show that $B_{0}$ has non trivial first order deformation, hence is not generic.

(iii) $A$ is a local non Scorza algebra. In dimension 5, there are exactly 5 isomorphism classes of such algebras. All but $E(4)$ have normal pattern $1 x y x^{2} x^{3}$, therefore deform via an interleaving of $K$ with a Scorza $(2,1)$-algebra (cf. [DP1]). We will study all the basisgraphs which occur; note that for every local non Scorza algebra of dimension 5, we build generally only one new algebra or family of the desired form which is not a fiber product (cf. Lemma 6).

Let $A=D^{4}$.

$B_{0}: e_{0}, e_{1} ; x, y, x^{2}, x^{3} \in e_{0} J e_{0} ; z_{1} \in e_{0} J e_{1}, z_{2} \in e_{1} J e_{0} ; y^{2}=$ $y x=x^{3}$.

$B: f_{0}, f_{1}, f_{2} ; u, v, u v \in f_{0} J^{\prime} f_{0} ; w_{1} \in f_{0} J^{\prime} f_{2} ; w_{2} \in f_{2} J^{\prime} f_{0}$.

The deformation is described as follows: by [DP1] IV.3.14 and IV.3.15, we normalize $D^{4}$ so that $x y=-y x=x^{3}$ and $y^{2}=-x^{3}$. By [DP1] IV.3.17, we get a deformation of $D^{4}$ to $\mathbf{K} \times F^{4}$ (where $F^{4}$ is the generic Scorza $(2,1)$ family, whose radical is generated by $u$ and $w)$. Add to the equations determining the deformation the following ones: $w_{1}=y_{1}, w_{2}=y_{2}, w_{1} w_{2}=u^{2}$; we get thus the desired deformation of the 8-dimensional algebra with mixed basisgraph given here.

Let $A=D_{c}^{5}$.

$B_{0}: e_{1}, e ; x, y, y^{2}, y^{3} \in e J e, z_{1} \in e J e_{1}, z_{2} \in e_{1} J e ; x^{2}=$ $z_{1} z_{2}=y^{3}$.

$B: f_{0}, f_{1}, f_{2} ; u, v, u v \in f_{1} J^{\prime} f_{1} ; w_{1} \in f_{1} J^{\prime} f_{0} ; w_{2} \in f_{0} J^{\prime} f_{1} ;$ $v u=w_{1} w_{2}=u v$.

The deformation is given by: $e=f_{1}+f_{2}, x=t\left(f_{1}-f_{2}\right)+u v$, $y=t\left(f_{1}-f_{2}\right)+u+v, z_{1}=w_{1}, z_{2}=w_{2}$.

If $A=D^{5}$, as $D^{5}$ deforms to $D^{4}$, by Lemma 6 we get a non generic case.

Let $A=D^{6}$. (1) As the loop $y$ is trivial, an algebra with weighted basis-graph $B_{0}: e_{0}, e_{1} ; x, y, x^{2}, x^{3} \in e_{1} J e_{1}, z_{1} \in e_{1} J e_{0}, z_{2} \in$ $e_{0} J e_{1} ; z_{1} z_{2}=x^{3}$ is deformable.

(2) The algebra with weighted basis-graph

$B_{0}: e_{0}, e_{1} ; x, y, x^{2}, x^{3} \in e_{1} J e_{1}, z_{1} \in e_{1} J e_{0}, z_{2} \in e_{0} J e_{1} ; z_{1} z_{2}=y$ is the fiber product of the 6-dimensional algebra with mixed basisgraph $(M 5)^{\prime \prime \prime}$ (see [DP1]) with the local 4-dimensional algebra $E(3)$ $\left(=\mathbf{K}[x] /\left(x^{4}\right)\right)$, which are both deformable; thus, our algebra is not 
generic. In these cases we recommend warmly that the reader draw the basis-graphs.

Let $A=E(4)$. $x^{4}$.

$B_{0}: e_{0}, e_{1} ; x, x^{2}, x^{3}, x^{4} \in e_{0} J e_{0}, y \in e_{0} J e_{1}, z \in e_{1} J e_{0} ; y z=$

$B: e_{0}^{\prime}, e_{1}^{\prime}, e_{1}^{\prime \prime} ; u, u^{2}, u^{3} \in e_{0}^{\prime} J^{\prime} e_{0}^{\prime}, w_{1} \in e_{0}^{\prime} J^{\prime} e_{1}^{\prime}, w_{2} \in e_{1}^{\prime} J e_{0}^{\prime} ;$ $w_{1} w_{2}=u^{3}$.

The deformation is given by: $e_{0}=e_{0}^{\prime}, e_{1}=e_{1}^{\prime}+e_{1}^{\prime \prime}, y=w_{1}$, $z=w_{2}$ and $x=u+t\left(e_{1}^{\prime}-e_{1}^{\prime \prime}\right)$,

The other candidates for generic algebras have basis-graph among those with either 2 idempotents or 3 idempotents. We now study them.

With two idempotents. We order the basis-graphs by the 2-type, always without mentioning the opposite one, as passing to the opposite 2-type corresponds to dualizing algebras, and we already said that an algebra is generic (resp. quasi-generic) if and only if its dual is so. These dual algebras and families will appear directly in the final results (cf. Appendices). The missing 2-types in this list are those for which we already used general methods in the previous pages. In the "status" column, we'll write $(*)$ for a generic algebra or family and (**) for a quasi-generic one.

$$
\begin{aligned}
& \frac{\text { 2-type }}{(0,3,0,3) \quad \frac{n^{0}}{(1)} \text { status }} \frac{\text { candidates } B_{0} \text { for genericity }}{e_{0}, e ; x, x^{2}, x^{3} \in e J e ;} \\
& y, y x, y x^{2} \in e_{0} J e \text {. } \\
& \text { (2) }(* *) \quad e_{0}, e_{1} ; x, y, x y \in e_{1} J e_{1} \text {, } \\
& z, z x, z y \in e_{0} J e_{1} ; y x=s x y \text {. }
\end{aligned}
$$

There are other algebras in Scorza $(2,1)$, but by Lemma 6, Propositions 1 and 2, or using trivial automorphisms, we eliminate all the remaining cases.

The weighted basis-graph (1) determines an algebra which deforms to an algebra $B$ with the following basis-graph:

$$
B: e_{0}, e_{1}, e_{2} ; v, v^{2} \in e_{2} J^{\prime} e_{2}, u, u v, u v^{2} \in e_{0} J^{\prime} e_{2} \text {. }
$$

where $e=e_{1}+e_{2}, x=(t / 2)\left(e_{1}-e_{2}\right)+u$ and $y=u$.

(2) determines a nongeneric family; a computer calculation indicates that for an algebra $A$ with this weighted basis-graph, the second cohomology group contains two first order deformations, one of them given by the following multiplication table: 


\begin{tabular}{|c|cccccc|}
\hline & $x_{2}$ & $x_{3}$ & $x_{4}$ & $x_{5}$ & $x_{6}$ & $x_{7}$ \\
\hline$x_{2}$ & & & & $\varepsilon x_{2}+x_{3}$ & $x_{4}$ & \\
$x_{3}$ & & & & & & \\
$x_{4}$ & & & & $\varepsilon \alpha x_{4}$ & & \\
$x_{5}$ & & & & $x_{5}$ & $x_{6}+x_{7}$ & \\
$x_{6}$ & & & & $\varepsilon \alpha x_{6}+r x_{7}$ & & \\
$x_{7}$ & & & & $\varepsilon \beta x_{7}$ & & \\
\hline
\end{tabular}

with $\alpha=(r+1) / 2$ and $\beta=(1-r) / 2$ and $\varepsilon^{2}=0$. We can easily verify that this multiplication is associative.

Let us now consider the structure constant tensor given by this multiplication table. The inner product of the deformation part of the structure constant tensor for an algebra with weighted basis-graph (2) with the deformation part of the structure constant tensor defined in the multiplication table above is non zero for general values of the parameters; hence the infinitesimal deformation given by the tensor is not trivial, and the given algebra is not generic, but, using a theorem of [DP-Sc2], we know that it is quasi-generic with $\operatorname{dim}$ Aut $A=10$.

For algebras with two idempotents, we will now omit the idempotents $e_{0}$ and $e_{1}$ in the presentation of $B_{0}$.

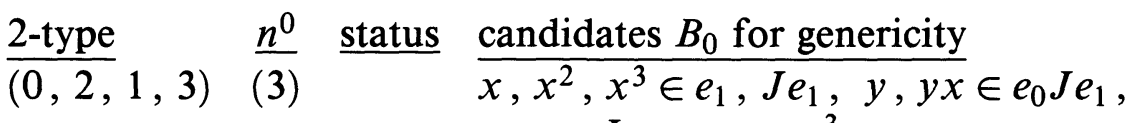

$$
\begin{aligned}
& z \in e_{1} J e_{0} ; z y=x^{3} \text {. } \\
& \text { (4) (**) } z_{1}, z_{2}, z_{1} z_{2} \in e_{1} J e_{1} \text {, } \\
& x, x z_{1} \in e_{0} J e_{1}, y \in e_{1} J e_{0} \text {; } \\
& z_{1} z_{2}=s z_{2} z_{1}, x z_{2}=x z_{1} \text {. } \\
& z_{1}, z_{2}, z_{1} z_{2} \in e_{1} J e_{1} \text {, } \\
& x, x z_{1} \in e_{0} J e_{1}, y \in e_{1} J e_{0} \text {; } \\
& z_{1} z_{2}=s z_{2} z_{1}, x z_{2}=0 \text {. } \\
& x, y \in e_{0} J e_{1}, z \in e_{1} J e_{0}, \\
& u, z x, z y \in e_{1} J e_{1} ; u^{2}=z y \text {. } \\
& x, x u \in e_{0} J e_{1}, y \in e_{1} J e_{0} \text {, } \\
& u, u^{2}, y x \in e_{1} J e_{1} \text {. }
\end{aligned}
$$

Note that some of the other weightings contain a trivial loop. Furthermore, Lemma 6 assures that these are the only relevant cases here. 
The weighted basis-graph (3) determines an algebra $B_{0}$ which deforms to the algebra $B$,

$B: e_{0}, e_{1}^{\prime}, e_{1}^{\prime \prime} ; u, u v \in e_{0} J^{\prime} e_{1}^{\prime}, w \in e_{1}^{\prime} J^{\prime} e_{0}$ and $v, w u \in e_{1}^{\prime} J e_{1}^{\prime}$ as follows: $e^{\prime}=e_{1}+e_{2}, x=(1 / t) v+\left(1 / t^{4}\right) w u+t^{2}\left(e_{1}^{\prime}-e_{1}^{\prime \prime}\right), y=t u$ and $z=(1 / t) w$.

(4) has a first order deformation, but no algebraic deformation, thus it is quasi-rigid ( $\operatorname{dim}$ Aut $=9$ ). A first order deformation is determined by the following equations: $x z_{1}=\varepsilon x, z_{1}^{2}=\varepsilon z_{1}, z_{1} z_{2}=$ $y x+\varepsilon[(s-1) / 2 s] z_{2}, z_{1}(y x)=\varepsilon[(s-1) / 2 s] y x,(y x) z_{1}=\varepsilon(y x)$ and $z_{1} y=\varepsilon[(s-1) / 2 s] y_{1}$, where $\varepsilon^{2}=0$.

(5) has a deformation to (4), given by $y^{\prime}=t y$.

(6) deforms to the algebra $B$ generated by

$B: e_{0}^{\prime}, e_{0}^{\prime \prime}, e_{1} ; x_{1}, y_{1} \in e_{1} J^{\prime} e_{0}^{\prime}, z_{1} \in e_{0}^{\prime} J^{\prime} e_{1}, z_{1} x_{1}, z_{1} y_{1} \in e_{0}^{\prime} J^{\prime} e_{0}^{\prime}$. by the following formulae: $e_{0}=e_{0}^{\prime}+e_{0}^{\prime \prime}, x=x_{1}, y=y_{1}, z=z_{1}$, $u=t\left(e_{0}^{\prime}-e_{0}^{\prime \prime}\right)+(1 / 2 t) z_{1} y_{1}$,

(7) has the following deformation:

$B: e_{0}^{\prime}, e_{0}^{\prime \prime}, e_{1} ; x_{1} \in e_{1} J^{\prime} e_{0}^{\prime \prime}, x_{2} \in e_{1} J^{\prime} e_{0}^{\prime}, y_{1} \in e_{0}^{\prime} J^{\prime} e_{1}, v \in e_{0}^{\prime \prime} J^{\prime} e_{0}^{\prime \prime}$, $y_{1} x_{2} \in e_{0}^{\prime} J^{\prime} e_{0}^{\prime}$

given by: $e_{0}=e_{0}^{\prime}+e_{0}^{\prime \prime}, u=t\left(e_{0}^{\prime}-e_{0}^{\prime \prime}\right)+(1 / 2 t) v, x=x_{2}+x_{1}, y=y_{1}$.

$\frac{2 \text {-type }}{(0,4,0,2)}$

$\underline{n^{0}}$ status candidates $B_{0}$ for genericity $x, y, x z, y z \in e_{0} J e_{1}, z, z^{2} \in e_{1} J e_{1}$.

$$
\begin{aligned}
& x, y, x z, x z^{2} \in e_{0} J e_{1}, z, z^{2} \in e_{1} J e_{1} ; \\
& y z=x z^{2} .
\end{aligned}
$$

$x, z, x y_{1}, x y_{2} \in e_{0} J e_{1}$,

$y_{1}, y_{2} \in e_{1} J e_{1} ; z y_{1}=x y_{2}$.

$x, z, x y_{1}, x y_{2} \in e_{0} J e_{1}$,

$y_{1}, y_{2} \in e_{1} J e_{1} ; z y_{1}=x y_{2}$.

By Proposition 3, we do not need to consider other cases. Other weightings reduce to the cases listed above by trivial automorphisms.

(8) deforms to the algebra $B$

$B: e_{0}, e_{1}^{\prime}, e_{1}^{\prime \prime} ; v \in e_{1}^{\prime \prime} J^{\prime} e_{1}^{\prime \prime}, u, z, u v, w v \in e_{0} J^{\prime} e_{1}^{\prime \prime}$. by: $e_{1}=e_{1}^{\prime}+e_{1}^{\prime \prime}, x=u, y=w, z=v+t\left(e_{1}^{\prime}-e_{1}^{\prime \prime}\right)$.

(9) deforms to the algebra $B$ generated by

$B: e_{0}, e_{1}^{\prime}, e_{1}^{\prime \prime} ; v \in e_{1}^{\prime \prime} J^{\prime} e_{1}^{\prime \prime}, w \in e_{0} J^{\prime} e_{1}^{\prime \prime}, u_{1}, u_{2}, u_{1} v \in e_{0} J^{\prime} e_{1}^{\prime \prime}$; $u_{1} v=u_{2} v$

by the following formulae: $e_{1}=e_{1}^{\prime}+e_{1}^{\prime \prime}, x=u_{1}+v, y=u_{2}$, $z=t\left(e_{1}^{\prime}-e_{1}^{\prime \prime}\right)+(1 / 2 t) v$. 
(10) deforms to the algebra $B$ generated by

$B: e_{0}, e_{1}^{\prime}, e_{1}^{\prime \prime} ; w \in e_{1}^{\prime} J^{\prime} e_{1}^{\prime}, u \in e_{0} J^{\prime} e_{1}^{\prime \prime}, v_{1}, v_{2}, v_{1} w \in e_{0} J^{\prime} e_{1}^{\prime}$; $v_{1} w=v_{2} w$

by the following formulae: $e_{1}=e_{1}^{\prime}+e_{1}^{\prime \prime}, x=u+v_{1}, z=v_{2}$, $y_{1}=t\left(e_{1}^{\prime}-e_{1}^{\prime \prime}\right)$ and $y_{2}=w$.

(11) deforms to (9) by: $y_{1}=t z, y_{2}=t z^{2}$ and $z^{\prime}=y$. (We get then $x y_{1}=t x z, x y_{2}=t x z^{2}, z^{\prime} y_{1}=y \cdot t z=t y z=t x z^{2}$ i.e. $z^{\prime} y_{1}=x y_{2}$.)

\begin{tabular}{|c|c|c|c|}
\hline \multirow[t]{6}{*}{$\frac{2 \text {-type }}{(0,3,1,2)}$} & $\frac{n^{0}}{(12)}$ & $\underline{\text { status }}$ & $\begin{array}{l}\text { candidates } B_{0} \text { for genericity } \\
y, y^{2} \in e_{1} J e_{1}, x, u, x y \in e_{0} J e_{1} \\
v \in e_{1} J e_{0} ; y^{2}=v u \text {. }\end{array}$ \\
\hline & (13) & & $\begin{array}{l}y, y^{2} \in e_{1} J e_{1}, x, x y, x y^{2} \in e_{0} J e_{1} \\
v \in e_{1} J e_{0} ; y^{2}=v u\end{array}$ \\
\hline & (14) & & $\begin{array}{l}x, x y, z_{1} \in e_{0} J e_{1}, z_{2} \in e_{1} J e_{0}, \\
y, y^{2} \in e_{1} J e_{1} .\end{array}$ \\
\hline & (15) & $(*)$ & $\begin{array}{l}x, y, y^{\prime} \in e_{0} J e_{1}, \quad z \in e_{1} J e_{0} \\
z x, z y \in e_{1} J e_{1}\end{array}$ \\
\hline & (16) & & $\begin{array}{l}x, x y, z_{1} \in e_{0} J e_{1}, z_{2} \in e_{1} J e_{0}, \\
y, z_{2} z_{1} \in e_{1} J e_{1} .\end{array}$ \\
\hline & (17) & & $\begin{array}{l}x, x y, x z \in e_{0} J e_{1}, u \in e_{1} J e_{0}, \\
y, z \in e_{1} J e_{1} .\end{array}$ \\
\hline
\end{tabular}

Any other weighting would contain a trivial loop, therefore would define an algebra with trivial idempotent-splitting deformation.

The weighted basis-graph (12) determines an algebra $B_{0}$ which deforms to:

$B: e_{0}, e_{1}^{\prime}, e_{1}^{\prime \prime} ; x_{1}, u_{1} \in e_{0} J^{\prime} e_{1}^{\prime \prime}, v_{1} \in e_{1}^{\prime \prime} J^{\prime} e_{0}, x_{2} \in e_{0} J^{\prime} e_{1}^{\prime}, v_{1} u_{1} \in$ $e_{1}^{\prime \prime} J^{\prime} e_{1}^{\prime \prime}$

by: $e_{1}=e_{1}^{\prime}+e_{1}^{\prime \prime}, x=x_{1}+x_{2}, u=u_{1}, v=v_{1}$ and $y=(t / 2)\left(e_{1}^{\prime \prime}-e_{1}^{\prime}\right)$ $+(1 / 2 t) v_{1} u_{1}$.

By Proposition 3, we get a deformation of (13) from a deformation of a 7-dimensional algebra (cf. [DP2], algebra $\left.(3)^{\prime}\right)$.

(14) and (16) both deform to the following basis-graph:

$B: e_{0}, e_{1}^{\prime}, e_{1}^{\prime \prime} ; u, v, v_{1} \in e_{0} J^{\prime} e_{1}^{\prime}, v_{2} \in e_{1}^{\prime} J^{\prime} e_{0}, v_{1} v_{2} \in e_{1}^{\prime} J^{\prime} e_{1}^{\prime}$. The deformation is given by $e_{1}=e_{1}^{\prime}+e_{1}^{\prime \prime}, x=u+v, z_{1}=v_{1}, z_{2}=v_{2}$ and either $y=t\left(e_{1}^{\prime}-e_{1}^{\prime \prime}\right)+(1 / 2 t) z_{2} z_{1}($ for $(14))$ or $y=t\left(e_{1}^{\prime}-e_{1}^{\prime \prime}\right)$ (for 
(16)). A deformation of (14) to (12) exists too.

(15) determines a rigid algebra $A$, as $H^{2}(A, A)=0$ (this result is given by a computer calculation). We get here one of the two promised examples, showing the importance of the hypothesis that the deformation be basic in Proposition 3. The dimension of the automorphism group is 12 .

By Proposition 3, we get deformations of (17) from a 7-dimensional algebra (cf. [DP2], algebra (3)").

\begin{tabular}{|c|c|c|c|}
\hline \multirow[t]{5}{*}{$\frac{\text { 2-type }}{(0,2,2,2)}$} & $\frac{n^{0}}{(18)}$ & $\underline{\text { status }}$ & $\begin{array}{l}\frac{\text { candidates } B_{0} \text { for genericity }}{x, x z \in e_{0} J e_{1} ; y, z y \in e_{1} J e_{0}} \\
z, z^{2} \in e_{1} J e_{1} ; y x=z^{2}\end{array}$ \\
\hline & (19) & $(*)$ & $\begin{array}{l}x, y \in e_{0} J e_{1} ; z, u \in e_{1} J e_{0} \\
z x, u y \in e_{1} J e_{1} .\end{array}$ \\
\hline & (20) & & $\begin{array}{l}x, x z \in e_{0} J e_{1} ; y, z y \in e_{1} J e_{0} \\
z, z^{2} \in e_{1} J e_{1}\end{array}$ \\
\hline & (21) & & $\begin{array}{l}x, x y_{1} \in e_{0} J e_{1} ; z, y_{2} z \in e_{1} J e_{0} \\
y_{1}, y_{2} \in e_{1} J e_{1} .\end{array}$ \\
\hline & (22) & & $\begin{array}{l}x, x z \in e_{0} J e_{1} ; y, z y \in e_{1} J e_{0} \\
z, y x \in e_{1} J e_{1}\end{array}$ \\
\hline
\end{tabular}

The other possibilities are eliminated either by Proposition 3 or by applying trivial automorphisms. Weightings containing a trivial loop are evidently not mentioned.

(19) determines a rigid algebra, because $H^{2}(A, A)=0$ (this result is given by a computer calculation). We get here the second promised example, similar to (15). The dimension of the automorphism group is equal to 8 .

The weighted basis-graph (18) determines an algebra $B_{0}$ which deforms to:

$B: e_{0}, e_{1}^{\prime}, e_{1}^{\prime \prime} ; x_{1} \in e_{0} J^{\prime} e_{1}^{\prime \prime} ; x_{2} \in e_{1}^{\prime} J^{\prime} e_{0} ; y_{1} \in e_{1}^{\prime \prime} J e_{0} ; y_{2} \in e_{0} J e_{1}^{\prime} ;$ $y_{1} x_{1} \in e_{1}^{\prime \prime} J^{\prime} e_{1}^{\prime \prime}$

by: $e_{1}=e_{1}^{\prime}+e_{1}^{\prime \prime}, z=t\left(e_{1}^{\prime \prime}-e_{1}^{\prime}\right)+(1 / 2 t) y_{1} x_{1}, x=x_{1}+x_{2}, y=y_{1}+y_{2}$.

An algebra $B_{0}$ with weighted basis-graph (20) deforms to:

$B: e_{0}, e_{1} ; v, v^{2} \in e_{1} J^{\prime} e_{1} ; u, u v \in e_{0} J^{\prime} e_{1} ; w, v w \in e_{1} J^{\prime} e_{0} ;$ $w u=v^{2}$

by: $x=t u, y=t w$ and $v=(1 / t) z$. Note that (20) has a deformation to the same algebra as (22) defined below, with $y=t\left(e_{1}^{\prime}-e_{1}^{\prime \prime}\right)+$ $(1 / 2 t) u_{1} v_{1}$. 
(21) deforms to:

$B: e_{0}, e_{1}^{\prime}, e_{1}^{\prime \prime} ; w \in e_{1}^{\prime \prime} J^{\prime} e_{1}^{\prime \prime}, v, v_{1}, v w \in e_{0} J^{\prime} e_{1}^{\prime \prime}, v_{2} \in e_{1}^{\prime} J^{\prime} e_{0}$ by: $e_{1}=e_{1}^{\prime}+e_{1}^{\prime \prime}, x=v, y_{1}=w, y_{2}=t\left(e_{1}^{\prime}-e_{1}^{\prime \prime}\right)$ and $z=v_{1}+v_{2}$.

(22) deforms to:

$B: e_{0}, e_{1}^{\prime}, e_{1}^{\prime \prime} ; u_{1} \in e_{0} J^{\prime} e_{1}^{\prime \prime}, u_{2} \in e_{0} J^{\prime} e_{1}^{\prime}, v_{1}, v_{2} \in e_{1}^{\prime} J^{\prime} e_{0}, u_{1} v_{1} \in$ $e_{1}^{\prime} J^{\prime} e_{1}^{\prime}$

with $e_{1}=e_{1}^{\prime}+e_{1}^{\prime \prime}, x=u_{1}+u_{2}, y=v_{1}+v_{2}, z=t\left(e_{1}^{\prime}-e_{1}^{\prime \prime}\right)$.

$$
\frac{\text { 2-type }}{(1,5,0,0) \quad \frac{n^{0}}{(23)} \quad \text { status }} \frac{\text { candidates } B_{0} \text { for genericity }}{x \in e_{0} J e_{0}, y, z, x y, x z, u \in e_{0} J e_{1}} \text {. }
$$

The other possible weightings can not appear, either because a trivial loop produces a trivial idempotent-splitting deformation, or as a consequence of Proposition 2. We have here a basis-graph of 2-type $(1,2 c+1,0,0)$; thus the algebra $B_{0}$ has an idempotent-splitting deformation, by Lemma 5 .

$$
\begin{aligned}
& \frac{\text { 2-type }}{(1,1,4,0)} \frac{n^{0}}{(24)} \quad \frac{\text { status }}{(*)} \quad \frac{\text { candidates } B_{0} \text { for genericity }}{y, y_{1}, y_{2}, y_{3}, y_{4} \in e_{1} J e_{0}} \\
& x \in e_{0} J e_{1}, x y \in e_{0} J e_{0} \text {. } \\
& y, y x, z, z x, y^{\prime} \in e_{1} J e_{0}, x \in e_{0} J e_{0}, \\
& w \in e_{0} J e_{1} \text {. }
\end{aligned}
$$

Applying trivial automorphisms, we can eliminate many other weightings; the remaining cases involve a trivial loop.

(24) determines a rigid algebra (a computer calculation indicates that the second Hochschild cohomology group is equal to 0 ). The dimension of the automorphism group is 10 .

(25) determines an algebra $B_{0}$ which deforms to

$B: e, e_{0}^{\prime}, e_{0}^{\prime \prime} ; u_{1}, u_{2} \in e J^{\prime} e_{0}^{\prime}, v_{1}, v_{2} \in e J^{\prime} e_{0}^{\prime \prime}, u_{3} \in e_{0}^{\prime} J^{\prime} e$ with $e_{1}=e, e_{0}=e_{0}^{\prime}+e_{0}^{\prime \prime}, x=(t / 2)\left(e_{0}^{\prime}-e_{0}^{\prime \prime}\right), y=u_{1}+v_{1}, z=u_{2}+v_{2}$ and $w=u_{3}$.

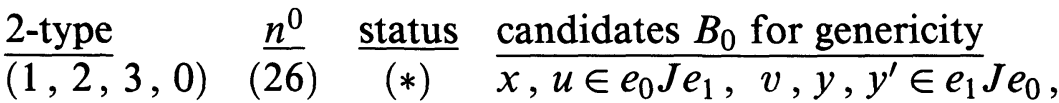

$$
\begin{aligned}
& u v=x y \in e_{0} J e_{0} \text {. } \\
& x \in e_{0} J e_{0}, y, x y \in e_{0} J e_{1} \text {, } \\
& z, z^{\prime}, z x \in e_{1} J e_{0} \text {. }
\end{aligned}
$$

Other weightings would involve a trivial loop, therefore the algebra would be trivially deformable. The remaining cases are eliminated by trivial automorphisms. 
(26) determine a rigid algebra (A computer calculation indicates that the second Hochschild cohomology group is equal to 0 ). The dimension of the automorphism group is 13 .

(27) determines an algebra $B_{0}$ which deforms to

$B: e, e_{0}^{\prime}, e_{0}^{\prime \prime} ; u_{1} \in e_{0}^{\prime} J^{\prime} e, u_{2}, u_{3} \in e J^{\prime} e_{0}^{\prime}, v_{1} \in e_{0}^{\prime \prime} J^{\prime} e, v_{2} \in e J^{\prime} e_{0}^{\prime \prime}$ with $e_{1}=e, e_{0}=e_{0}^{\prime}+e_{0}^{\prime \prime}, x=(t / 2)\left(e_{0}^{\prime}-e_{0}^{\prime \prime}\right), y=u_{1}+v_{1}, z=u_{2}+v_{2}$ and $w=u_{3}$.

$$
\begin{aligned}
& \frac{\text { 2-type }}{(1,4,0,1)} \frac{n^{0}}{(28)} \quad \text { status } \quad \frac{\text { candidates } B_{0} \text { for genericity }}{x \in e_{0} J e_{0}, z \in e_{1} J e_{1},} \\
& y, y z, x y, x y z \in e_{0} J e_{1} \text {. }
\end{aligned}
$$

Every other weighting, but one, contains either a trivial loop or a trivial arrow; the non genericity is trivial in the first case, and is a consequence of Proposition 3 in the second one. The algebra $B_{0}$ determined by (28) deforms to

$B: e_{0}^{\prime}, e_{1}^{\prime}, e_{2}^{\prime} ; v \in e_{0}^{\prime} J^{\prime} e_{0}^{\prime}, u, u v \in e_{1}^{\prime} J^{\prime} e_{0}^{\prime}, w, w v \in e_{1}^{\prime \prime} J^{\prime} e_{0}$ where $e_{0}=e_{1}^{\prime}+e_{2}^{\prime}, e_{1}=e_{0}^{\prime}, x=t\left(e_{1}^{\prime}-e_{2}^{\prime}\right), y=u+w$ and $z=v$ (we get $x y=t(u-w), y z=u v+w v$ and $x y z=t(u v-w v)$ ).

$$
\begin{aligned}
& \frac{\text { 2-type }}{(1,3,1,1)} \frac{n^{0}}{(29)} \quad \frac{\text { status }}{(*)} \quad \frac{\text { candidates } B_{0} \text { for genericity }}{x, y, x z y \in e_{0} J e_{1}, z \in e_{1} J e_{0}}, \\
& x z \in e_{0} J e_{0}, z y \in e_{1} J e_{1} \text {. } \\
& x, x y, u \in e_{0} J e_{1}, v \in e_{1} J e_{0} \text {, } \\
& u v \in e_{0} J e_{0}, y \in e_{1} J e_{1} \text {. } \\
& x \in e_{0} J e_{0}, z \in e_{1} J e_{1}, \\
& y, x y, y z \in e_{0} J e_{1}, y^{\prime} \in e_{1} J e_{0} \text {. }
\end{aligned}
$$

Every other weighting contains either a trivial loop or a trivial arrow; the non genericity is trivial in the first case, and is a consequence of Proposition 3 in the second one. The remaining case reduces to (24) by a trivial automorphism.

As a computer calculation indicates that the second Hochschild cohomology group is 0 , the algebra determined by $(28)$ is rigid, hence generic, with $\operatorname{dim}$ Aut $=9$.

(30) determines an algebra $B_{0}$ which deforms to

$B_{0}: e_{0}, e_{1}^{\prime}, e_{1}^{\prime \prime} ; z \in e_{0} J^{\prime} e_{1}^{\prime \prime}, w, u_{1} \in e_{0} J^{\prime} e_{1}^{\prime}, v_{1} \in e_{1}^{\prime} J^{\prime} e_{0}, u_{1} v_{1} \in$ $e_{0} J^{\prime} e_{0}$

by $e_{1}=e_{1}^{\prime}+e_{1}^{\prime \prime}, x=w+z, y=t\left(e_{1}^{\prime}-e_{1}^{\prime \prime}\right), u=u_{1}$ and $v=v_{1}$.

By Proposition 3, (31) has an idempotent-splitting deformation, hence is not generic. 


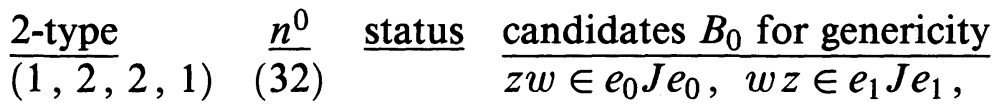

$$
\begin{aligned}
& z, z w z \in e_{0} J e_{1}, w, w z w \in e_{1} J e_{0} \text {. } \\
& x \in e_{0} J e_{0}, z \in e_{1} J e_{1}, \\
& y, x y \in e_{0} J e_{1}, u, z u \in e_{1} J e_{0} \text {. } \\
& x \in e_{0} J e_{0}, z y \in e_{1} J e_{1} \text {, } \\
& y, x y \in e_{0} J e_{1}, z, z^{\prime} \in e_{1} J e_{0} \text {. }
\end{aligned}
$$

By Proposition 3, there is no other case to consider here.

(32) has the following deformations, the first one being of Flanigan's type II, the second one of Flanigan's type I; (see [Fl]):

$$
M_{2}(\mathbf{K}) \times M_{2}(\mathbf{K}) \rightarrow B=M_{2}(\mathbf{K}[\varepsilon]) \rightarrow B_{0} .
$$

Denote by $E_{11}, E_{22}$ the idempotents and by $E_{12}, E_{21}$ the solid arrows in $B$. The actual deformation of $A$ to $B$ is given by: $z=$ $t E_{12}+y_{12}$ and $w=t E_{21}+y_{21}$. Remember that $y_{12}=x_{1} E_{12}=E_{12} x_{2}$ and $y_{21}=x_{2} E_{21}=E_{21} x_{1}$. We get then $z w=t^{2} e_{1}+2 t x_{1}$ and $z w z=$ $t^{3} E_{12}+3 t^{2} y_{12}, w z=t^{2} e_{2}+2 t x_{2}$ and $w z w=t^{3} E_{21}+3 t^{2} y_{21}$.

(33) determines an algebra $B_{0}$ which deforms to

$B: e_{0}, e_{1}^{\prime}, e_{1}^{\prime \prime} ; x_{1} \in e_{0} J_{0}^{\prime}, w \in e_{1}^{\prime \prime} J_{0}^{\prime}, y_{1}, x_{1} y_{1} \in e_{0} J^{\prime} e_{1}^{\prime}, v \in$ $e_{1}^{\prime} J^{\prime} e_{0}$

by: $e_{1}=e_{1}^{\prime}+e_{1}^{\prime \prime}, x=x_{1}, y=y_{1}, z=t\left(e_{1}^{\prime}-e_{1}^{\prime \prime}\right)$ and $u=v+w$.

(34) deforms in a similar way; note that the non genericity in this case is also a consequence of Proposition 3.

$$
\begin{aligned}
\frac{n^{0}}{(2,3,0,1) \quad \text { stape }}(35) \quad & \frac{\text { candidates } B_{0} \text { for genericity }}{x, x^{2} \in e_{0} J e_{0}, z \in e_{1} J e_{1},} \\
& y, x y, x^{2} y \in e_{0} J e_{1} ; y z=x^{2} y . \\
& x, x^{2} \in e_{0} J e_{0}, z \in e_{1} J e_{1}, \\
& y, x y, y z \in e_{0} J e_{1} .
\end{aligned}
$$

The other weightings give us either fiber products of a 6-dimensional algebra with mixed basis-graph with $\operatorname{Scorza}(1,1)$, or algebras containing a trivial loop, or algebras containing a trivial arrow.

(35) determines an algebra $B_{0}$ which deforms to

$B: f_{01}, f_{02}, f_{03}, f_{11}, f_{12} ; z \in f_{02} J^{\prime} f_{02}, u_{1} \in f_{01} J^{\prime} f_{11}, u_{2}, z u_{2} \in$ $f_{02} J^{\prime} f_{12}$,

by: $e_{0}=f_{03}+f_{02}+f_{01}, e_{1}=f_{12}+f_{11}, x=(t / 2)\left(f_{02}-f_{01}\right)+z$, $y^{\prime}=u_{2}+u_{1}$. 
(36) determines an algebra $B_{0}$ which deforms to

$B: e_{0}, e_{1}^{\prime}, e_{1}^{\prime \prime} ; u, u^{2} \in e_{0} J^{\prime} e_{0}, w \in e_{0} J^{\prime} e_{1}^{\prime}, v, u v \in e_{0} J^{\prime} e_{1}^{\prime \prime}$ by: $e_{1}=e_{1}^{\prime}+e_{1}^{\prime \prime}, z=t\left(e_{1}^{\prime}-e_{1}^{\prime \prime}\right), y=v+w$ and $x=u$.
$\frac{2 \text {-type }}{(2,2,1,1)}$
$\frac{n^{0}}{(37)}$
status $\frac{\text { candidates } B_{0} \text { for genericity }}{x z, y z \in e_{0} J e_{0}, z x \in e_{1} J e_{1}}$
$x, y \in e_{0} J e_{1}, z \in e_{1} J e_{0}$.
(38)
$x, x^{2} \in e_{0} J e_{0}, z y \in e_{1} J e_{1}$,
$y, x y \in e_{0} J e_{1}, z y \in e_{1} J e_{0} ; y z=x^{2}$.
$x, x^{2} \in e_{0} J e_{0}, z y \in e_{1} J e_{1}$,
$y, x y \in e_{0} J e_{1}, z \in e_{1} J e_{0}$.
$x, y u \in e_{0} J e_{0}, \quad z \in e_{1} J e_{1}$,
$y, x y \in e_{0} J e_{1}, \quad u \in e_{1} J e_{0}, x y=y z$

$$
\begin{aligned}
& x, x^{2} \in e_{0} J e_{0}, \quad z \in e_{1} J e_{1}, \\
& y, x y \in e_{0} J e_{1}, u \in e_{1} J e_{0} ; y z=x y, \\
& y u=x^{2} .
\end{aligned}
$$

Other weightings are eliminated, either by Proposition 3 or by the presence of a trivial loop.

(37) determines an algebra $B_{0}$ which deforms to

$B: e_{0}^{\prime}, e_{0}^{\prime \prime}, e_{1}^{\prime}, e_{1}^{\prime \prime} ; x_{2}^{\prime} \in e_{0}^{\prime} J^{\prime} e_{1}^{\prime}, x_{3}^{\prime} \in e_{0}^{\prime \prime} J^{\prime} e_{1}^{\prime}, x_{4}^{\prime} \in e_{1}^{\prime} J^{\prime} e_{0}^{\prime}, x_{5}^{\prime} \in$ $e_{0}^{\prime \prime} J^{\prime} e_{0}^{\prime}$

with $e_{0}=e_{0}^{\prime}+e_{0}^{\prime \prime}, e_{1}=e_{1}^{\prime}+e_{1}^{\prime \prime}, x_{2}=t x_{2}^{\prime}, x_{3}=x_{3}^{\prime}, x_{4}=t x_{4}^{\prime}$, $x_{5}=t x_{5}^{\prime}, x_{6}=x_{5}^{\prime}+(1 / 2) t^{2}\left(e_{0}^{\prime}-e_{0}^{\prime \prime}\right)$ and $x_{7}=\left(1 / t^{2}\right)\left(e_{1}^{\prime}-e_{1}^{\prime \prime}\right)$.

(38) determines an algebra $B_{0}$ which deforms to

$B: e_{0}^{\prime}, e_{0}^{\prime \prime}, e_{1} ; y_{3} \in e_{0}^{\prime} J^{\prime} e_{1}, y_{4} \in e_{1} J^{\prime} e_{0}^{\prime}, y_{5} \in e_{0}^{\prime \prime} J^{\prime} e_{1}, y_{6} \in e_{0}^{\prime} J^{\prime} e_{0}^{\prime}$, $y_{7} \in e_{1} J^{\prime} e_{1}$

where $e_{0}=e_{0}^{\prime}+e_{0}^{\prime \prime}, x_{2}=t\left(e_{0}^{\prime}-e_{0}^{\prime \prime}\right)+(1 / 2 t) y_{6}, x_{7}=y_{7}, x_{3}=y_{3}+y_{5}$, $x_{4}=y_{4}, x_{5}=y_{6}$ and $x_{6}=t\left(y_{3}-y_{5}\right)$.

(39) determines an algebra $B_{0}$ which deforms to

$B: e_{0}^{\prime}, e_{0}^{\prime \prime}, e_{1} ; u \in e_{0}^{\prime} J^{\prime} e_{0}^{\prime}, v, u v \in e_{0}^{\prime} J^{\prime} e_{1}, w \in e_{1} J^{\prime} e_{0}^{\prime}, w v \in$ $e_{1} J^{\prime} e_{1}$

by: $e_{0}=e_{0}^{\prime}+e_{0}^{\prime \prime}, x=t\left(e_{0}^{\prime}-e_{0}^{\prime \prime}\right)+(1 / t) u, y=t v, z=(1 / t) w$. A deformation of (39) to (38) exists too (Flanigan's type II, cf. [FI]).

(40) determines an algebra $B_{0}$ which deforms to

$B: e_{0}^{\prime}, e_{0}^{\prime \prime}, e_{1} ; v_{1}, v_{1} w \in e_{0}^{\prime} J^{\prime} e_{1}, v_{2} \in e_{1} J^{\prime} e_{0}^{\prime}, v_{1} v_{2} \in e_{0}^{\prime} J^{\prime} e_{0}^{\prime}$, $w \in e_{1} J^{\prime} e_{1}$ 
by $e_{0}=e_{0}^{\prime}+e_{0}^{\prime \prime}, x=t\left(e_{0}^{\prime}-e_{0}^{\prime \prime}\right)+v_{1} v_{2}, y=v_{1}+(1 / t) v_{1} w, z=w$ and $u=v_{2}$.

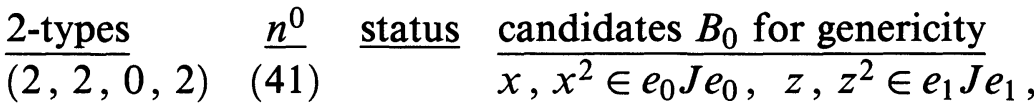

$$
\begin{aligned}
& y, x y \in e_{0} J e_{1}, y z=x y \text {. } \\
& x, x^{2} \in e_{0} J e_{0}, z, z^{2} \in e_{1} J e_{1}, \\
& y, x y \in e_{0} J e_{1} \text {. }
\end{aligned}
$$

The other weightings are eliminated either by the one-way bridge corollary or because of the presence of a trivial loop.

(41) determines an algebra $B_{0}$ which deforms to

$B: e_{0}^{\prime}, e_{0}^{\prime \prime}, e_{1} ; u, v u=u w \in e_{0}^{\prime} J^{\prime} e_{1}^{\prime}, v \in e_{0}^{\prime} J^{\prime} e_{0}^{\prime}, w \in e_{1}^{\prime} J^{\prime} e_{1}^{\prime}$ by $e_{0}=e_{0}^{\prime}+e_{0}^{\prime \prime}, e_{1}=e_{1}^{\prime}+e_{1}^{\prime \prime}, x=t\left(e_{0}^{\prime}-e_{0}^{\prime \prime}\right)+v, y=u$ and $z=t\left(e_{1}^{\prime}-e_{1}^{\prime \prime}\right)+w$.

(42) determines an algebra $B_{0}$ which deforms to

$B: e_{0}, e_{1}^{\prime}, e_{1}^{\prime \prime} ; u, u^{2} \in e_{0} J^{\prime} e_{0}, v, u v \in e_{0} J^{\prime} e_{1}^{\prime}, w \in e_{1}^{\prime} J^{\prime} e_{1}^{\prime}$

by the following formulae: $e_{1}=e_{1}^{\prime}+e_{1}^{\prime \prime}, x=u, y=v, z=w+$ $t\left(e_{1}^{\prime}-e_{1}^{\prime \prime}\right)$. Note that the algebra we consider here is the fiber product of a 6-dimensional non generic algebra with mixed basic-graph (cf. [DP1], p. 62, the algebra (M3) $\left.{ }^{\prime \prime \prime}\right)$ with $\mathbf{K}[z] /\left(z^{3}\right)$, which deforms to $\mathbf{K} \times \mathbf{K}[w] /\left(w^{2}\right)$.

$\frac{2 \text {-types }}{(2,1,1,2)}$
$\underline{n^{0}}$
status candidates $B_{0}$ for genericity
$x, x^{2}=y z \in e_{0} J e_{0}, u, u^{2}=z y \in e_{1} J e_{1}$, $y \in e_{0} J e_{1}, z \in e_{1} J e_{0}$.
$x, x^{2}=y z \in e_{0} J e_{0}, u, u^{2} \in e_{1} J e_{1}$, $y \in e_{0} J e_{1}, z \in e_{1} J e_{0}$.
$x, x^{2} \in e_{0} J e_{0}, u, u^{2} \in e_{1} J e_{1}$,
$y \in e_{0} J e_{1}, z \in e_{1} J e_{0}$.

Every other weighting contains at least one trivial loop, defining thus an algebra with an idempotent-splitting deformation.

(43) determines an algebra $B_{0}$ which deforms to

$B_{0}: e_{0}^{\prime}, e_{0}^{\prime \prime}, e_{1}^{\prime}, e_{1}^{\prime \prime} ; v \in e_{0}^{\prime} J^{\prime} e_{1}^{\prime}, w \in e_{1}^{\prime} J^{\prime} e_{0}^{\prime}, v w \in e_{0}^{\prime} J^{\prime} e_{0}^{\prime}, w v \in$ $e_{1}^{\prime} J^{\prime} e_{1}^{\prime}$

by $e_{0}=e_{0}^{\prime}+e_{0}^{\prime \prime}, e_{1}=e_{1}^{\prime}+e_{1}^{\prime \prime}, y=v, z=w, x=t\left(e_{0}^{\prime}-e_{0}^{\prime \prime}\right)+$ $(1 / 2 t) v w, u=t\left(e_{1}^{\prime}-e_{1}^{\prime \prime}\right)+(1 / 2 t) w v$.

(44) determines an algebra $B_{0}$ which deforms to

$B: e_{0}, e_{1}^{\prime}, e_{1}^{\prime \prime} ; u, u^{2} \in e_{0} J^{\prime} e_{0}, v_{1} \in e_{0} J^{\prime} e_{1}^{\prime}, v_{2} \in e_{1}^{\prime} J^{\prime} e_{0}, w \in$ $e_{1}^{\prime} J^{\prime} e_{1}^{\prime}$ 
by: $e_{1}=e_{1}^{\prime}+e_{1}^{\prime \prime}, x=u, y=w+t\left(e_{1}^{\prime}-e_{1}^{\prime \prime}\right), z_{1}=v_{1}$ and $z_{2}=v_{2}$.

(45) determines an algebra $B_{0}$ which deforms to

$B: e_{0}^{\prime}, e_{0}^{\prime \prime}, e_{1}^{\prime}, e_{1}^{\prime \prime} ; u \in e_{0} J^{\prime} e_{0}, v_{1} \in e_{0} J^{\prime} e_{1}^{\prime}, v_{2} \in e_{1}^{\prime} J^{\prime} e_{0}, w \in$ $e_{1}^{\prime} J^{\prime} e_{1}^{\prime}$

by: $e_{0}=e_{0}^{\prime}+e_{0}^{\prime \prime}, e_{1}=e_{1}^{\prime}+e_{1}^{\prime \prime}, x=u+t\left(e_{0}^{\prime}-e_{0}^{\prime \prime}\right), y=w+t\left(e_{1}^{\prime}-e_{1}^{\prime \prime}\right)$, $z_{1}=v_{1}, z_{2}=v_{2}$. Note that deformations of (44) and (45) to (43) exist too (Flanigan's type II, of [Fl]).

$$
\begin{aligned}
& \frac{\text { 2-types }}{(3,2,0,1)} \frac{n^{0}}{(46)} \quad \text { status } \frac{\text { candidates } B_{0} \text { for genericity }}{x, x^{2}, x^{3} \in e_{0} J e_{0}, z \in e_{1} J e_{1}} \text {, } \\
& y, y z \in e_{0} J e_{1} ; x y=y z \text {. } \\
& \text { (*) } \quad x, y, x y \in e_{0} J e_{0}, z \in e_{1} J e_{1} \text {, } \\
& u, x u \in e_{0} J e_{1} ; y x=\sigma x y \text {; } \\
& u z=x u ; y u=x u \text {. }
\end{aligned}
$$

Every other weighting falls into one of the following cases: (1) the fiber product of two algebras, at least one of them having an idempotentsplitting deformation; (2) a weighted basis-graph containing a trivial loop.

$$
\frac{\text { 2-types }}{(3,1,1,1) \quad \frac{n^{0}}{(48)} \quad \text { status }} \begin{aligned}
& \frac{\text { candidates } B_{0} \text { for genericity }}{x, x^{2}, x^{3}=y z \in e_{0} J e_{0}, z y} \in e_{1} J e_{1}, \\
& y \in e_{0} J e_{1}, z \in e_{1} J e_{0} .
\end{aligned}
$$

(*) $\quad x, y, x y \in e_{0} J e_{0}, u z \in e_{1} J e_{1}$, $z \in e_{0} J e_{1}, u \in e_{1} J e_{0} ; x y=z u$, $y x=s x y$.

Every other weighting either defines a fiber-product of two algebras, at least one of them having an idempotent-splitting deformation, or contains a trivial loop.

(46) determines an algebra $B_{0}$ which deforms to

$B: e_{0}^{\prime}, e_{0}^{\prime \prime}, e_{1} ; u, u^{2} \in e_{0}^{\prime} J^{\prime} e_{0}^{\prime}, v, u v=v w \in e_{0}^{\prime} J^{\prime} e_{1}, w \in e_{1} J^{\prime} e_{1}$ by: $e_{0}=e^{\prime}+e^{\prime \prime}, x=u+t\left(e^{\prime}-e^{\prime \prime}\right), y=v, w=z$.

By [DP1] III.5.1 Proposition 8 and a computer calculation, (47) and (49) define semi-rigid (thus generic) families (dim Aut $=7$ for both families).

(48) determines an algebra $B_{0}$ which deforms to

$B: e_{0}^{\prime}, e_{0}^{\prime \prime}, e_{1} ; u, u^{2}=v w \in e_{0}^{\prime} J^{\prime} e_{0}^{\prime}, v \in e_{0}^{\prime} J^{\prime} e_{1}, w \in e_{1} J e_{0}^{\prime}$, $w v \in e_{1} J^{\prime} e_{1}$

by: $e_{0}=e_{0}^{\prime}+e_{0}^{\prime \prime}, x=t\left(e_{0}^{\prime}-e_{0}^{\prime \prime}\right)+(1 / t) u+u^{2}, y=v, z=w$. 
With three idempotents-without cycles. Most of the basis-graphs appearing here determine algebras which are fiber products of a deformable algebra along loopless idempotents either with the arrow algebra $T$ or with the 4-dimensional two reversed arrow algebra. We will list here only the special cases, again without mentioning the three idempotents $e_{0}, e_{1}$ and $e_{2}$.

$\frac{n^{0}}{(50)} \quad \frac{\text { status }}{(*)} \frac{\text { candidates } B_{0} \text { for genericity }}{x \in e_{0} J e_{1}, y \in e_{1} J e_{0}, x y \in e_{0} J e_{0},}$ $z_{1}, z_{2}$ between $e_{1}$ and $e_{2}$.

(59) (**) $\quad x, y, x y \in e_{0} J e_{0}, z$ between $e_{1}$ and $e_{0}$, $z^{\prime}$ between $e_{1}$ and $e_{2} ; y x=s x y$

( $s$ different from $-1,0,1)$.

(60) (**) $\quad z$ between $e_{0}$ and $e_{1}, z^{\prime}$ between $e_{1}$ and $e_{2}$, $x, y, x y \in e_{1} J e_{1} ; y x=s x y(s$ different from $-1,0,1)$.

(61) $\quad(*) \quad x y \in e_{0} J e_{0}, x, x^{\prime} \in e_{0} J e_{1}, y \in e_{0} J e_{1}$, $z$ between $e_{1}$ and $e_{2}$. 
By computer calculations we get that $(50)$ determines three rigid algebras (dim Aut $=10$ for two of them, 8 for the third one) and (61) determines two rigid algebras (dim Aut $=9$ ). By application of Lemma 1, we get that (59) and (60) determine quasi-generic families, with $\operatorname{dim} A u t=6$. A deformation of $(58)$ is described in [Sc2]. Furthermore, by Lemma 1, we know that (51), (52), (56), (57) and (58) are nongeneric.

An algebra $B_{0}$ with basis-graph (53) deforms to

$B: e_{0}, e_{1}, e_{2}, e_{3} ; u_{0} \in e_{0} J^{\prime} e_{2}, v_{0} \in e_{0} J^{\prime} e_{3}, u_{1} \in e_{2} J^{\prime} e_{1}, v_{1} \in$ $e_{3} J^{\prime} e_{1}$

by $e^{\prime}=e_{2}+e_{3}, z=t\left(e_{2}-e_{3}\right), x=u_{0}+v_{0}, y=u_{1}+v_{1}$.

Especially interesting is the deformation of $B_{0}$ with basis-graph (54) to $M_{2}(\mathbf{K}) \times M_{2}(\mathbf{K})$, with matrix units $E_{i j}$ and $E_{i j}^{\prime}(i, j \in\{0,1\})$ as follows: $e_{0}=E_{00}, e_{2}=E_{11}+E_{00}^{\prime}, e_{1}=E_{11}^{\prime}, x=t E_{10}, y=t E_{01}$, $z=E_{01}^{\prime}, u=t E_{10}^{\prime}$.

(55) is the fiber product of a 5-dimensional algebra with mixed basis-graph with the 4-dimensional Kronecker algebra. As every 5dimensional algebra with mixed basis-graph is deformable, so is (56). Actually, this algebra deforms the product of $M_{2}(\mathbf{K})$ with the 4dimensional Kronecker algebra.

With three idempotents-with cycles.
$\underline{n^{0}} \quad \underline{\text { status }}$
(62)

$$
\begin{gathered}
x \in e_{0} \frac{\text { candidates } B_{0} \text { for genericity }}{J e_{0}, y, x y \in e_{0} J e_{1}, \quad z \in e_{1}} J e_{2}, \\
y z \in e_{0} J e_{2} . \\
x y \in e_{0} J e_{0}, \quad x \in e_{0} J e_{1}, y \in e_{1} J e_{0}, \\
z \in e_{1} J e_{2}, x_{2} \in e_{0} J e_{2} . \\
x y \in e_{0} J e_{0}, \quad x \in e_{0} J e_{1}, y \in e_{1} J e_{0}, \\
y z \in e_{1} J e_{2}, \quad z \in e_{0} J e_{2} .
\end{gathered}
$$$$
x, x^{2} \in e_{0} J e_{0}, y, y^{\prime}, y^{\prime \prime} \text { form a triangle. }
$$

An algebra $B_{0}$ with basis-graph (62) deforms to

$B: e_{0}, e_{1}, e_{2}, e_{3} ; v \in e_{2} J^{\prime} e_{1}, w \in e_{1} J^{\prime} e_{0}, v w \in e_{2} J^{\prime} e_{0}, u \in$ $e_{1} J e_{3}$

by $e^{\prime}=e_{2}+e_{3}, x=t\left(e_{2}-e_{3}\right), y=v-u, z=w$. (We get $x y=t(v+u)$ and $y z=v w$.) 
An algebra $B_{0}$ with basis-graph (63) deforms to

$B$ : matrix units $E_{00}, E_{11}, E_{01}, E_{10}$; idempotents $E_{2}, E_{3}$; arrows $x \in E_{00} J^{\prime} E_{2}, y \in E_{11} J^{\prime} E_{2}$ by $e_{1}=E_{11}, e_{2}=E_{2}, e_{0}=E_{00}+E_{3}, x=t E_{01}, y=t E_{10}, z=v$.

(64) has a deformation, in a similar way. Finally, by [DP1], Corollary III.7.1, (65) defines a non generic algebra.

With four idempotents. There are four possible cases, each of them including a trivial loop; therefore, all the corresponding algebras are non generic.

As in [DP2], we denote by $p(n)$ the number of reduced irreducible components of $\operatorname{Alg}_{n}$ and by $q(n)$ the total number of irreducible components, including the non reduced ones. Recall that the number of irreducible components dominated by algebras with loopless basis-graph is equal to 136; they are all reduced (for a complete list, see [DP1]). Adding the results of the present section, we have the following proposition:

Proposition 16. Alg $_{n}$ contains at least 164 reduced irreducible components, each of them dominated by the algebras with weighted basis-graph either loopless or mixed; in the last case, they are listed in Appendix I. Furthermore, there are at least 20 quasi-generic algebras or families, listed in Appendix II.

$$
p(8) \geq 164 ; q(8) \geq 184 \text {. }
$$

\section{Appendix I. Generic mixed basis-graphs of dimension 8.}

$\operatorname{dim}$ Aut $A$

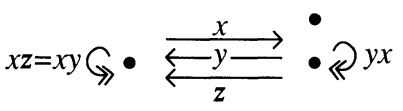

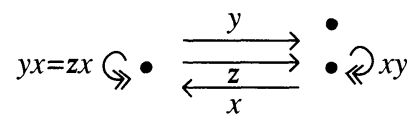

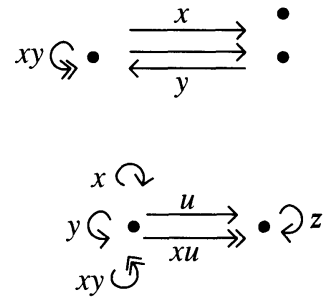

$(y x=s x y ; u z=x u ; y u=x u)$
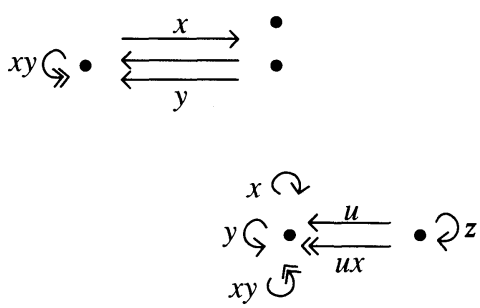

$(y x=s x y ; u x=z u ; u y=u x)$ 
$\operatorname{dim}$ Aut $A$

$$
\begin{aligned}
& y G \stackrel{\frac{z}{\longleftarrow}}{\longleftarrow} \cdot \partial u z \\
& x y=z u \circlearrowleft \quad u \quad(y x=s x y)
\end{aligned}
$$

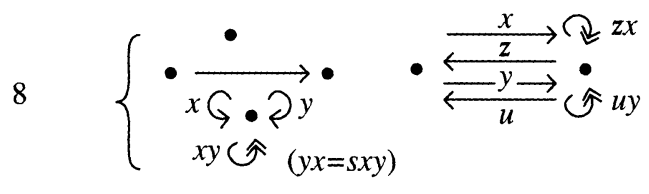

$$
\begin{aligned}
& x y G \cdot \frac{x}{\longleftrightarrow} \cdot \stackrel{\longrightarrow}{\longleftrightarrow} \\
& x y \mathrm{G}_{\rightarrow} \cdot \frac{x}{\longleftarrow}
\end{aligned}
$$

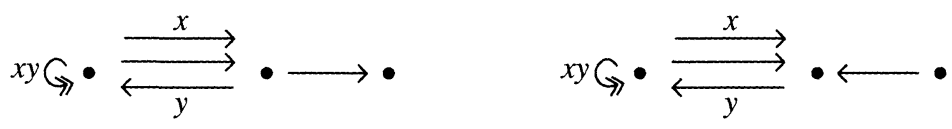

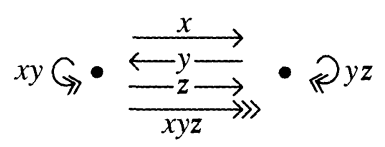

10
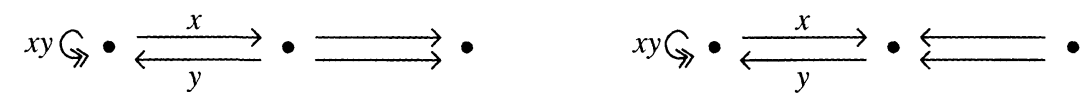

12

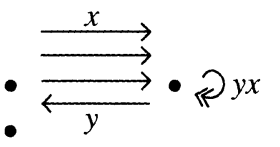

- $\frac{x}{\longleftarrow}$

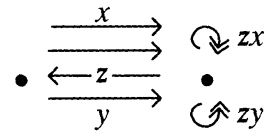

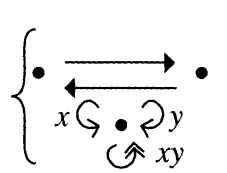

$(y x=s x y)$

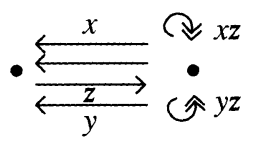

13

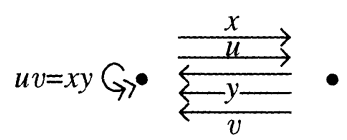

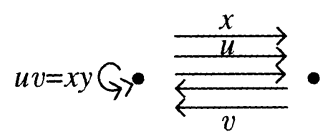

19
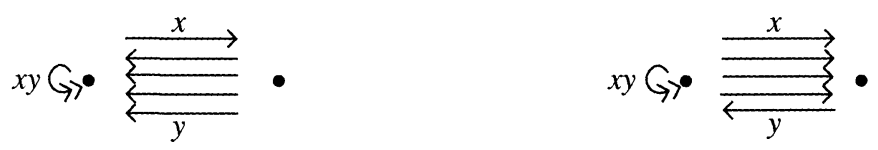

$$
\left\{\begin{array}{l}
\cdot \frac{x}{x \overrightarrow{G \cdot \partial y}} \bullet \\
x y \hat{G} \quad(y x=s x y)
\end{array}\right.
$$


Appendix II. Known quasi-generic mixed basis-graphs of dimension 8.

$\operatorname{dim}$ Aut $A$

5
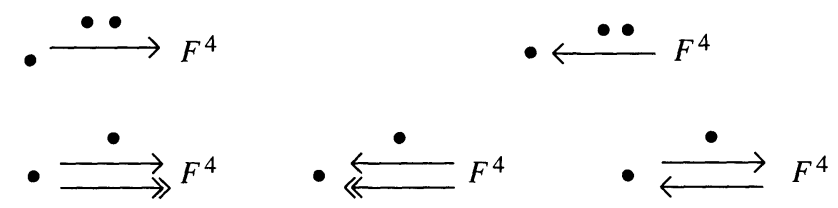

6

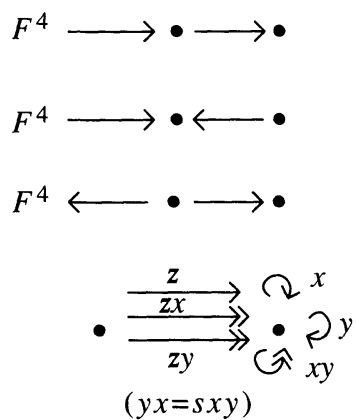

$\bullet \longrightarrow F^{4} \longrightarrow \bullet \quad F^{4} \longleftarrow \bullet \longleftarrow$

$\bullet \longleftarrow F^{4} \longrightarrow \bullet$

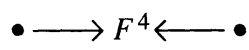

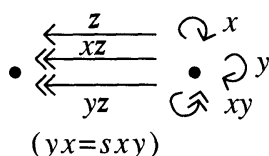

$\bullet \longrightarrow$ Scorza $(4,1)$

$\bullet \longleftarrow \operatorname{Scorza}(4,1)$

7

- $\longrightarrow$ Scorza $(3,2)$

- $\longleftarrow$ Scorza $(3,2)$

9

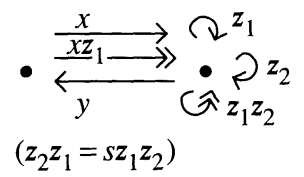

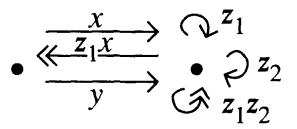
$\left(z_{2} z_{1}=s z_{1} z_{2}\right)$

Notation. $F^{4}=K\langle x, y\rangle /\left(x^{2}, y^{2}, y x-\sigma x y\right), \sigma$ different from $1,-, 0$. 


\section{Appendix Ib. Generic mixed basis-graphs of dimension 8.}

\begin{tabular}{|c|c|c|c|}
\hline $\operatorname{dim}$ Aut $A$ & Idempotents & Filtered radical basis & Relations \\
\hline$\# 6$ & $e_{0}, e_{1}, e_{2}$ & $\begin{array}{l}x \in e_{1} J e_{2} \\
y, z \in e_{2} J e_{1} \\
x y \in e_{1} J e_{1}, y z \in e_{2} J e_{2}\end{array}$ & $x z=x y$ \\
\hline \#7 & $\begin{array}{l}e_{0}, e_{1} \\
e_{2}, e_{3}\end{array}$ & $\begin{array}{l}x, x^{\prime} \in e_{1} J e_{2}, y \in e_{2} J e_{1} \\
x y \in e_{1} J e_{1}\end{array}$ & \\
\hline$\# 7$ & $e_{0}, e_{1}$ & $\begin{array}{l}x, y, x y \in e_{0} J e_{0}, z \in e_{1} J e_{1} \\
u, x u \in e_{0} J e_{1}\end{array}$ & $\begin{array}{l}y x=s x y ; u z=x u \\
y u=x u\end{array}$ \\
\hline 7 & $e_{0}, e_{1}$ & $\begin{array}{l}x, y, x y \in e_{0} J e_{0}, \quad z \in e_{0} J e_{1} \\
u \in e_{1} J e_{0}, u z \in e_{1} J e_{1}\end{array}$ & $\begin{array}{l}z u=x y \\
y x=s x y\end{array}$ \\
\hline 8 & $\begin{array}{l}e_{0}, e_{1} \\
e_{2}, e_{3}\end{array}$ & $\begin{array}{l}x, y, x y \in e_{0} J e_{0} \\
z \in e_{1} J e_{2}\end{array}$ & $y x=s x y$ \\
\hline 8 & $e_{0}, e_{1}, e_{2}$ & $\begin{array}{l}x \in e_{0} J e_{1}, y \in e_{1} J e_{0} \\
u \in e_{1} J e_{2}, v \in e_{2} J e_{1} \\
x y \in e_{0} J e_{0}\end{array}$ & \\
\hline 8 & $e_{0}, e_{1}$ & $\begin{array}{l}x, y \in e_{0} J e_{1}, z, u \in e_{1} J e_{0} \\
z x, u y \in e_{1} J e_{1}\end{array}$ & \\
\hline \#9 & $e_{0}, e_{1}, e_{2}$ & $\begin{array}{l}x \in e_{0} J e_{1}, y, y^{\prime} \in e_{1} J e_{0} \\
z \in e_{1} J e_{2}, x y \in e_{0} J e_{0}\end{array}$ & \\
\hline$\# 9$ & $e_{0}, e_{1}, e_{2}$ & $\begin{array}{l}x \in e_{0} J e_{1}, y, y^{\prime} \in e_{1} J e_{0} \\
z \in e_{2} J e_{1}, x y \in e_{0} J e_{0}\end{array}$ & \\
\hline 9 & $e_{0}, e_{1}$ & $\begin{array}{l}x, z, x y z \in e_{0} J e_{1}, y \in e_{1} J e_{0} \\
x y \in e_{0} J e_{0}, y z \in e_{1} J e_{1}\end{array}$ & \\
\hline \#10 & $e_{0}, e_{1}$ & $\begin{array}{l}x \in e_{0} J e_{1}, \quad y \in e_{1} J e_{0} \\
u, v \in e_{1} J e_{2}, x y \in e_{0} J e_{0}\end{array}$ & \\
\hline \#12 & $e_{0}, e_{1}, e_{2}$ & $\begin{array}{l}x_{1}, x_{2}, x_{3} \in e_{0} J e_{1} \\
x_{4} \in e_{1} J e_{0} \\
x_{4} x_{1} \in e_{1} J e_{1}\end{array}$ & \\
\hline 12 & $e_{0}, e_{1}, e_{2}$ & $\begin{array}{l}x_{1}, x_{2} \in e_{0} J e_{1} \\
x_{3}, x_{4} \in e_{1} J e_{0} \\
x_{4} x_{1} \in e_{1} J e_{1}\end{array}$ & \\
\hline 12 & $e_{0}, e_{1}, e_{2}$ & $M_{2}(K) \times F^{4}$ & \\
\hline$\# 12$ & $e_{0}, e_{1}, e_{2}$ & $\begin{array}{l}x_{1}, x_{2}, x_{4} \in e_{0} J e_{1} \\
x_{3} \in e_{1} J e_{0} \\
x_{3} x_{1}, x_{3} x_{2} \in e_{1} J e_{1}\end{array}$ & \\
\hline$\# 13$ & $e_{0}, e_{1}$ & $\begin{array}{l}x_{1}, x_{2} \in e_{0} J e_{1} \\
x_{3}, x_{4}, x_{5} \in e_{1} J e_{0} \\
x_{1} x_{3} \in e_{0} J e_{0}\end{array}$ & $x_{2} x_{4}=x_{1} x_{3}$ \\
\hline$\# 19$ & $e_{0}, e_{1}$ & $\begin{array}{l}x_{1} \in e_{0} J e_{1}, x_{1} x_{2} \in e_{0} J e_{0} \\
x_{2}, x_{3}, x_{4}, x_{5} \in e_{1} J e_{0}\end{array}$ & \\
\hline 24 & $e_{0}, e_{1}, e_{2}$ & $\begin{array}{l}x_{1}, x_{2} \in e_{0} J e_{1} \\
x_{3}, x_{4}, x_{3} x_{4} \in e_{2} J e_{2}\end{array}$ & $x_{3} x_{4}=s x_{4} s x_{3}$ \\
\hline
\end{tabular}

N.B. 1) The parameter $s$ is always different from $-1,0,1$.

2) The symbol \# means that the dual algebra has to be counted too.

Total: 28 generic algebras or families. 
Appendix IIb. Known quasi-generic mixed basis-graphs of dimension 8.

\begin{tabular}{|c|c|c|c|}
\hline $\operatorname{dim}$ Aut $A$ & Idempotents & Filtered radical basis & Relations \\
\hline$\# 5$ & $\begin{array}{l}e_{0}, e_{1} \\
e_{2}, e_{3}\end{array}$ & $\begin{array}{l}x_{1} \in e_{0} J e_{1} \\
x_{2}, x_{3} \in e_{1} J e_{1} \\
x_{2} x_{3} \in e_{1} J e_{1}\end{array}$ & $x_{2} x_{3}=s x_{3} x_{2}$ \\
\hline$\# 5$ & $e_{0}, e_{1}, e_{2}$ & $\begin{array}{l}x_{1}, x_{1} x_{3} \in e_{0} J e_{1} \\
x_{3}, x_{4}, x_{3} x_{4} \in e_{1} J e_{1}\end{array}$ & $\begin{array}{l}x_{4} x_{3}=s x_{3} x_{4} \\
x_{1} x_{4}=x_{1} x_{3}\end{array}$ \\
\hline 5 & $e_{0}, e_{1}, e_{2}$ & $\begin{array}{l}x_{1} \in e_{0} J e_{1} \\
x_{2} \in e_{1} J e_{0} \\
x_{3}, x_{4}, x_{3} x_{4} \in e_{1} J e_{1}\end{array}$ & $\begin{array}{l}x_{4} x_{3}=s x_{3} x_{4} \\
x_{2} x_{1}=x_{3} x_{4}\end{array}$ \\
\hline \#6 & $e_{0}, e_{1}, e_{2}$ & $\begin{array}{l}x_{1}, x_{2}, x_{1} x_{2} \in e_{0} J e_{0} \\
x_{3} \in e_{0} J e_{1} \\
x_{4} \in e_{1} J e_{2}\end{array}$ & $x_{2} x_{1}=s x_{1} x_{2}$ \\
\hline 6 & $e_{0}, e_{1}, e_{2}$ & $\begin{array}{l}x_{1} \in e_{0} J e_{1} \\
x_{2}, x_{3}, x_{2} x_{3} \in e_{1} J e_{1} x_{4} \in e_{1} J e_{2}\end{array}$ & $x_{3} x_{2}=s x_{2} x_{3}$ \\
\hline \#6 & $e_{0}, e_{1}, e_{2}$ & $\begin{array}{l}x_{1}, x_{2}, x_{1} x_{2} \in e_{0} J e_{0} \\
x_{3} \in e_{0} J e_{1} \\
x_{4} \in e_{2} J e_{1}\end{array}$ & $x_{2} x_{1}=s x_{1} x_{2}$ \\
\hline \#6 & $e_{0}, e_{1}, e_{2}$ & $\begin{array}{l}x_{1} \in e_{0} J e_{1} \\
x_{2}, x_{3}, x_{2} x_{3} \in e_{1} J e_{1} \\
x_{4} \in e_{2} J e_{1}\end{array}$ & $x_{3} x_{2}=s x_{2} x_{3}$ \\
\hline$\# 6$ & $e_{0}, e_{1}$ & $\begin{array}{l}x_{1}, x_{1} x_{2} \in e_{0} J e_{1} \\
x_{2}, x_{3}, x_{2} x_{3} \in e_{1} J e_{1}\end{array}$ & $x_{3} x_{2}=s x_{2} x_{3}$ \\
\hline$\# 6$ & $e_{0}, e_{1}$ & $\begin{array}{l}x_{1} \in e_{0} J e_{1} \\
x_{2}, x_{3}, x_{4}, x_{5} \in e_{1} J e_{1} \\
x_{2} x_{3} \in e_{1} J e_{1}\end{array}$ & $\begin{array}{l}x_{3} x_{2}=s x_{2} x_{3} \\
x_{4} x_{5}=t x_{2} x_{3} \\
x_{5} x_{4}=x_{2} x_{3} \\
\end{array}$ \\
\hline \#7 & $e_{0}, e_{1}$ & $\begin{array}{l}x_{1} \in e_{0} J e_{1} \\
x_{2}, x_{3}, x_{4} \in e_{1} J e_{1} \\
x_{3} x_{2}, x_{4} x_{2} \in e_{1} J e_{1}\end{array}$ & $\begin{array}{l}x_{4}^{2}=x_{3} x_{2}+\mu_{1} x_{4} x_{2} \\
x_{3}^{2}=\mu_{2} x_{3} x_{2}+x_{4} x_{2} \\
x_{2} x_{4}=\mu_{3} x_{4} x_{2} \\
x_{2} x_{3}=\mu_{4} x_{3} x_{2} \\
x_{2}^{2}=\mu_{5} x_{3} x_{2}+\mu_{6} x_{4} x_{2}\end{array}$ \\
\hline$\# 9$ & $e_{0}, e_{1}$ & $\begin{array}{l}x_{1}, x_{1} x_{3} \in e_{0} J e_{1} \\
x_{2} \in e_{1} J e_{0} \\
x_{3}, x_{4}, x_{3} x_{4} \in e_{1} J e_{1}\end{array}$ & $\begin{array}{l}x_{2} x_{1}=x_{3} x_{4} \\
x_{4} x_{3}=s x_{3} x_{4}\end{array}$ \\
\hline
\end{tabular}

N.B. The symbol \# indicates that the opposite algebra has to be considered here too. We get the basis-graph by reversing the arrows.

\section{REFERENCES}

[DP1] T. Dana-Picard, Classifying generic algebras, Ph.D. Thesis, Bar-Ilan University, 1990.

[DP2] _ 7-dimensional algebras with mixed basis-graph, Canad. Math. Soc. Conf. Proc., Vol. 11 (1991), 123-158. 
[DP-Sc1] T. Dana-Picard and M. Schaps, Classifying generic algebras, Rocky Mountain J. Math., $22 \mathrm{n}^{\circ} 1$ (1992), 125-155.

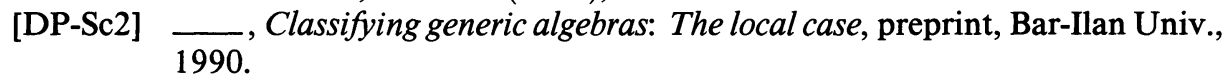

[DP-Sc3] _ Non reduced components of $\mathbf{A l g}_{n}$, preprint, Bar-Ilan Univ., 1990.

[DP-Sc4] _ A computer assisted project: Classification of algebras, to appear in Proc. of Int. Conf. on Computational Algebra and Algebraic Geometry, Cortona 91, Cambridge Acad. Press.

[Fl] F. Flanigan, Which algebras deform to a total matrix algebra?, J. Algebra, 299 (1974), 103-112.

[Ga] P. Gabriel, Finite representation type is open, in Representations of Algebras, LNM 488, Springer-Verlag, 1974, 132-155.

[Ge] M. Gerstenhaber, On the deformations of rings and algebras, Ann. of Math., $79 \mathrm{n}^{\circ} 1$ (1964), 59-63.

[Ha] D. Happel, Deformations of five dimensional algebras with unit, in Ring Theory, LN in Pure and Applied Math., 51, Dekker, New York, 1978, 453-494.

[Kr] H. Kraft, Geometric Methods in Representation Theory, LNM 944, Springer-Verlag, 1980, 180-258.

[Ma] G. Mazzola, The algebraic and geometric classification of associative algebras of dimension 5, Manuscripta Math., 27 (1979), 81-101.

[Sc1] M. Schaps, Deformations of finite dimensional algebras and their idempotents, Trans. Amer. Math. Soc., $307 \mathrm{n}^{\circ} 2$ (1988), 843-856.

[Sc2] _ Deformations of algebras and Hochschild cohomology II, Perspectives in Ring Theory, NATO ASI Series C, vol. 233, Kluwer, Dordrecht, $1988,41-58$.

Received February 3, 1992 and in revised form, September 15, 1992. This work is a part of the author's Ph.D. thesis at Bar-Ilan University. Partially supported by Israel Academy of Science.

BEN GURION UNIVERSITY

BeER SheVA 84105, IsRael

Current address: Bar-Ilan University

Ramat Gan 52900, Israel

E-mail address: dana@bimacs.bitnet 



\section{CONTENTS}

K. Auinger, Free products of combinatorial strict inverse semigroups . . . . . . . . 201

T. Dana-Picard, Generic 8-dimensional algebras with mixed basis-graph . . . . . . 229

D. Dimovski, One-parameter fixed point indices . . . . . . . . . . . . . . . . . 263

K. S. Druschel, Oriented orbifold cobordism . . . . . . . . . . . . . . . . . . . 299

A. D. Helfer, Conjugate points on spacelike geodesics or pseudo-self-adjoint MorseSturm-Liouville systems . . . . . . . . . . . . . . . . . . . . . 321

J. G. Heywood and O. D. Walsh, A counter-example concerning the pressure in the Navier-Stokes equations, as $t \rightarrow 0^{+} \ldots \ldots \ldots \ldots \ldots \ldots \ldots \ldots \ldots, \ldots \ldots \ldots$

G. Masbaum and P. Vogel, 3-valent graphs and the Kauffman bracket . . . . . . 361

R. Stong, The Jones polynomial of parallels and applications to crossing number 383

Vol. 164, No. 2 


\section{PACIFIC JOURNAL OF MATHEMATICS}

Volume $164 \quad$ No. $2 \quad$ June 1994

Free products of combinatorial strict inverse semigroups

201

KARL AUINGER

Generic 8-dimensional algebras with mixed basis-graph

229

THIERRY DANA-PICARD

One-parameter fixed point indices

263

DONCO DIMOVSKI

Oriented orbifold cobordism

299

KIMBERLY SUE DRUSCHEL

Conjugate points on spacelike geodesics or pseudo-self-adjoint

Morse-Sturm-Liouville systems

ADAM D. HELFER

A counterexample concerning the pressure in the Navier-Stokes

351 equations, as $t \rightarrow 0^{+}$

JOHN GROVES HeYwOOD and OWEN WALSH

3-valent graphs and the Kauffman bracket

Gregor Masbaum and PierRe Vogel

The Jones polynomial of parallels and applications to crossing number 383

RICHARD ANDREW STONG 